\title{
Storm-time ring current: model-dependent results
}

\author{
N. Yu. Ganushkina ${ }^{1,2}$, M. W. Liemohn ${ }^{1}$, and T. I. Pulkkinen ${ }^{3}$ \\ ${ }^{1}$ Department of Atmospheric, Oceanic and Space Sciences, University of Michigan, Ann Arbor, MI, USA \\ ${ }^{2}$ Finnish Meteorological Institute, Helsinki, Finland \\ ${ }^{3}$ Aalto University, School of Electrical Engineering, Espoo, Finland
}

Correspondence to: N. Yu. Ganushkina (natalia.ganushkina@fmi.fi)

Received: 18 July 2011 - Revised: 15 November 2011 - Accepted: 6 January 2012 - Published: 17 January 2012

\begin{abstract}
The main point of the paper is to investigate how much the modeled ring current depends on the representations of magnetic and electric fields and boundary conditions used in simulations. Two storm events, one moderate (SymH minimum of $-120 \mathrm{nT}$ ) on 6-7 November 1997 and one intense (SymH minimum of $-230 \mathrm{nT}$ ) on 21-22 October 1999 , are modeled. A rather simple ring current model is employed, namely, the Inner Magnetosphere Particle Transport and Acceleration model (IMPTAM), in order to make the results most evident. Four different magnetic field and two electric field representations and four boundary conditions are used. We find that different combinations of the magnetic and electric field configurations and boundary conditions result in very different modeled ring current, and, therefore, the physical conclusions based on simulation results can differ significantly. A time-dependent boundary outside of $6.6 R_{\mathrm{E}}$ gives a possibility to take into account the particles in the transition region (between dipole and stretched field lines) forming partial ring current and near-Earth tail current in that region. Calculating the model SymH* by Biot-Savart's law instead of the widely used Dessler-Parker-Sckopke (DPS) relation gives larger and more realistic values, since the currents are calculated in the regions with nondipolar magnetic field. Therefore, the boundary location and the method of SymH* calculation are of key importance for ring current data-model comparisons to be correctly interpreted.
\end{abstract}

Keywords. Magnetospheric physics (Current systems; Energetic particles, trapped; Storms and substorms)

\section{Introduction}

The ring current is a key current system in the Earth's magnetosphere. It encircles the Earth in the region of $R=2-7 R_{\mathrm{E}}$ $\left(R_{\mathrm{E}}=6371 \mathrm{~km}\right)$ and is carried mainly by energetic ions and electrons in the energy range from 30 to $300 \mathrm{keV}$. Farther away $\left(R>8 R_{\mathrm{E}}\right)$, the plasma sheet has a characteristic ion temperature of only a few $\mathrm{keV}$. The ring current is a defining element of magnetic storms. It dominates the energy content of the inner magnetospheric plasma, and therefore is the primary factor in altering the electric and magnetic fields in this region of geospace.

Extensive observational and modeling studies of the ring current properties have been made during more than 50 years of space era. There exist several models for the ring current. The most extensively used are the modifications of RAM (Ring current-Atmosphere interactions Model) developed initially by Fok et al. (1993) and Jordanova et al. (1996). There are now, at least, 5 variations of this model currently in use for the magnetospheric physics studies (e.g., Fok et al., 2003; Jordanova et al., 2006; Khazanov et al., 2004; Zheng et al., 2006; Liemohn et al., 2006), and every version has been developed in its own way during the last 20 years. These models solve the gyration and bounce averaged kinetic equation for the main hot particle species $\left(\mathrm{H}^{+}, \mathrm{O}^{+}, \mathrm{He}^{+}\right.$, and electrons) in the $\mathrm{keV}$ energy range. There are three more models (Chen et al., 1993; Ebihara and Ejiri, 2000; Ganushkina et al., 2005), where the particle motion is followed in the drift approximation, and the Liouville theorem is used for particle flux calculations. Most of the models include Coulomb collisional scattering and decay, precipitation loss to the upper atmosphere, and charge exchange loss.

Another widely used inner magnetosphere model is the Rice Convection Model (RCM), which describes plasma electrodynamics in the inner and middle magnetosphere and its coupling to the ionosphere (Wolf et al., 1982; Spiro et al., 1981). The RCM represents the plasma distribution in terms of multiple fluids. Its equations and numerical methods have been specifically designed for treatment of the inner magnetosphere, including the flow of electric currents along magnetic field lines to and from the conducting ionosphere. The model computes these currents and the associated electric fields self-consistently. The recently developed 
Rice Convection Model Equilibrium (RCM-E) (Toffoletto et al., 2003; Lemon et al., 2003) combines the drift physics and magnetosphere-ionosphere coupling computational machinery of the RCM with a model of equilibrium magnetic field in static force balance with the RCM-computed pressures.

All of the ring current models set up the modeling region and define the boundary conditions. They also set up the configuration of the electric and magnetic fields, in which the particles move, by using the background representations for these fields, or calculate them self-consistently, or combination of both. Previous studies mainly used different convection electric fields for the modeling of ring current formation and development during storms. The magnetic field was usually assumed to be a dipole. Simulations were performed for many storms (Kozyra et al., 1998; Ebihara and Ejiri, 2000; Jordanova et al., 2001; Liemohn et al., 2002, 2006). In these calculations several representations of the electric field were used such as (1) Volland-Stern large-scale convection field (Volland, 1973; Stern, 1975), (2) modified McIlwain field (McIlwain, 1986; Liemohn et al., 2001), (3) Boyle et al. (1997) polar cap potential applied for the intensity of the convection, (4) ionospheric electric potential patterns obtained by Weimer (1996), and (5) a more complex electric field configurations based on the assimilative mapping of ionospheric electrodynamics (AMIE) (Richmond, 1992) with the addition of a penetration electric field. The ionospheric electric potential obtained with the AMIE procedure involves the synthesis of ground-based and satellite data. Recently, to simulate ring current development during high speed streams, (Jordanova et al., 2009) used the newly developed University of New Hampshire Inner Magnetospheric Electric Field (UNH-IMEF) from Cluster data for the inner magnetospheric convection.

Angelopoulos et al. (2002) conducted the testing of global storm-time electric field representations, such as VollandStern, and modifications of Weimer ionospheric potentials, using particle spectra on POLAR, EQUATOR-S, and FAST spacecraft. They found that significant differences with ion spectral observations exist and cannot be accounted for simply by modification of existing representations.

Another method of specifying the electric field is to calculate it self-consistently with the hot plasma solution (e.g., Jaggi and Wolf, 1973). Recently, self-consistent descriptions of ring current ions and the magnetospheric electric field taking into account the large scale magnetosphere-ionosphere electrodynamic coupling was introduced in Comprehensive Ring Current Model (CRCM) (Fok et al., 2001), and selfconsistent RAM model (Ridley and Liemohn, 2002). These models calculate the inner magnetospheric potential electric field self-consistently, proceeding from a prescribed potential on the outer boundary. Liemohn et al. (2006) conducted several plasmasphere-ring current simulations and data comparisons for two magnetic storms. They found that their nominal self-consistent electric field was the best at reproducing the selected data.
In contrast, not many studies have been devoted to the role of the magnetic field to the ring current evolution. The dipole field approximation has often been considered to be reasonable, based on the assumption that the Earth's magnetic field is only slightly disturbed in the inner magnetosphere (Gamayunov et al., 2009). A dipole approximation, however, excludes many important physical processes, such as drift shell splitting. Moreover, the ring current buildup during storms produces a magnetic field in the opposite direction to the background magnetic field. This can modify the magnetic field significantly (Tsyganenko et al., 2003) and also the storm-time particle trajectories. The recent study by Ganushkina et al. (2010) has shown that during the main phase of even a moderate storm, the nondipole magnetic field contribution can be as big as $30 \%$ of the dipole value at $4 R_{\mathrm{E}}$ and $80 \%$ at $6 R_{\mathrm{E}}$ at midnight. The distortion of the magnetic field during storms was also modeled by Zaharia et al. (2005). It stresses once again the importance of realistic magnetic field for the ring current modeling.

Ganushkina et al. (2006) followed the evolution of the proton ring current during a storm using (1) the dipole, (2) the Tsyganenko T89 (Tsyganenko, 1989), and the (3) storm-time Tsyganenko T01S (Tsyganenko, 2002) magnetic field representations. They found that changing the magnetic field from dipole to realistic decreases the ring current energy content by about $30 \%$.

Jordanova et al. (2006) included a more realistic, selfconsistent, magnetic field treatment. The anisotropic pressure calculated with the RAM ring current model was used as input for a 3-D equilibrium code by Zaharia et al. (2004) and the force-balanced magnetic field was computed at 1$\mathrm{h}$ time intervals. The procedure of RAM coupling with the 3-D equilibrium code and the resulting self-consistent magnetic field results were presented by Zaharia et al. (2006). The newly calculated magnetic field (interpolated between the 1-h intervals) was then used in RAM to update the particle drifts. They found $\sim 50 \%$ decrease in the ring current pressure compared to the dipole field calculation. The largescale morphology of the ring current fluxes was not affected significantly and the local time of the equatorial flux peaks remained almost unchanged. Chen et al. (2006) also found similar decreases in the pressure of tens of percent with magnetically self-consistent ring current calculations.

Fok et al. (2001) solved the kinetic equation in an Euler potential space on the ionosphere, obtaining the distribution in the equatorial plane by mapping along prescribed realistic magnetic field lines. Ebihara et al. (2008) have extended the comprehensive ring current model (CRCM) (Fok et al., 2001) to incorporate a self-consistent magnetic field in the inner magnetosphere, named extended CRCM (ECRCM). Their method iteratively updates the magnetic field, obtained by solving the Biot-Savart equation. In the latest development of the radiation belts model, Fok et al. (2008) have used the Tsyganenko and Sitnov TS04 configuration (Tsyganenko and Sitnov, 2005) for the magnetic field. 
Since the plasma sheet is a very important source of the ring current populations, using realistic particle boundary conditions is critical for accurate ring current modeling. For proper modeling, all source population characteristics have to be considered, namely, the radial location, MLT dependence, and spectrum of the source. There have been a number of investigations to study that. For example, Kozyra et al. (1998); Chen et al. (2000) and Ebihara et al. (2005) have shown that plasma sheet density is of key importance to a strong ring current. Ebihara et al. (2005) investigated the nonlinear impact of the plasma sheet density on the total energy of the storm-time ring current. Observations (Thomsen et al., 2003; Lavraud et al., 2006; Liemohn et al., 2008) and ring current simulations (Chen et al., 2007; Lavraud and Jordanova, 2007) have shown that a cold dense plasma sheet leads to an enhanced ring current. Zheng et al. (2010) have shown that local time distribution of the source plays an important role in determining both the radial and azimuthal (local time) location of the ring current peak pressure. Also, their simple modeling has shown that a source that is farther away from the Earth leads to a stronger ring current than a source that is closer to the Earth.

The Dst index is a global measure of the low-latitude magnetic perturbation quantifying the distortion of the Earth's magnetic field (Sugiura and Kamei, 1991). 1 min SymH index can also be used. Dst index was thought to be well correlated with the ring current energy density from storm maximum well into recovery (Hamilton et al., 1988; Greenspan and Hamilton, 2000). The Dessler-Parker-Sckopke relationship (Dessler and Parker, 1959; Sckopke, 1966) (hereinafter referred to as DPS) relates the total energy content of the plasma within the inner magnetosphere to a magnetic perturbation at the center of the Earth. This perturbation is a rough equivalent to the Dst index if it is assumed (Carovillano and Siscoe, 1973) that it is close to the perturbation averaged around the equator of the Earth.

Several studies, however, have suggested that the Dst index contains contributions from many other sources than the azimuthally symmetric ring current (Campbell, 1973; Arykov and Maltsev, 1993; Dremukhina et al., 1999; Alexeev et al., 2001; Ohtani et al., 2001; Liemohn, 2003; Ganushkina et al., 2004; Kalegaev et al., 2005). Based on GOES 8 measurements and their correlation with Dst, Ohtani et al. (2001) determined the contribution from the tail current at Dst minimum to be, at least, $25 \%$. Liemohn (2003) showed that this is roughly accounted for by a systematic overestimation inherent in the DPS relation (because of the assumption that all of the plasma is within the integration volume, the DPS relation implicitly includes a truncation current). By modeling several storm events, Ganushkina et al. (2004) have shown that the tail current intensifies first and tracks the drop in the Dst index. The ring current develops more slowly, and then stays at an increased level longer than the tail current. During moderate storms (Dst about $-150 \mathrm{nT}$ ), both ring and tail currents are intensified, the tail current contributes more to Dst than the ring current. On the other hand, during intense storms (Dst $<-200 \mathrm{nT}$ ), the tail current is intensified, and remains nearly constant, while the ring current follows the Dst variations. Thus, the information contained in the Dst index is different during small and large storms.

Usually in the ring current models, the outer boundary is set at $6.6 R_{\mathrm{E}}$, where plasma density and temperature observations are available from the LANL geostationary satellites (Bame et al., 1993). These measurements can then be used to determine the boundary conditions in the plasma sheet (Jordanova et al., 2001; Liemohn et al., 2001; Ganushkina et al., 2006). The particles inside the geostationary orbit are identified as the ring current particles. As a result, the Dst minimum is significantly underestimated during storm-time ring current modeling (see, for example, Jordanova et al., 2006).

A complementary approach to ring current modeling was made by Milillo et al. (2001), who used data from the Charge-Energy-Mass instrument (CHEM) onboard AMPTE/CCE (Active Magnetospheric Particle Tracer Explorers/Charge Composition Explorer) satellite to formulate an empirical model for the average equatorial $\mathrm{H}^{+}$fluxes in the inner magnetosphere. The model gives a parameterization for the ion distributions, and time-dependent tuning of the model parameters then gives the global evolution of the proton distribution in the equatorial magnetosphere. Orsini et al. (2004) demonstrated that the parameter variations can be associated with convection, injection, and diffusion processes. In particular, the model empirically identified the diffusion-associated high-energy population, which increases in energy with decreasing distance from the Earth.

As was mentioned above, many studies were conducted using the ring current simulations where different combinations of different representations for magnetic and electric fields and boundary conditions were used, and a number of conclusions were made on the physics of the storm-time ring current. In the present paper we attempt to investigate how much the modeled ring current depends on the configurations on fields and boundary conditions used in simulations and, correspondingly, how much of the physical conclusions made from simulations depend on them too. We employ a rather simple ring current model, namely, the Inner Magnetosphere Particle Transport and Acceleration model (IMPTAM), developed by Ganushkina et al. (2001, 2005, 2006), in order to make the results most evident. We use four different magnetic field and two electric field representations and four boundary conditions in this study. The main questions we are going to answer are:

1. How does the choice of the

(a) magnetic field,

(b) electric field, and

(c) boundary conditions (time-dependence, spectrum and radial location) 

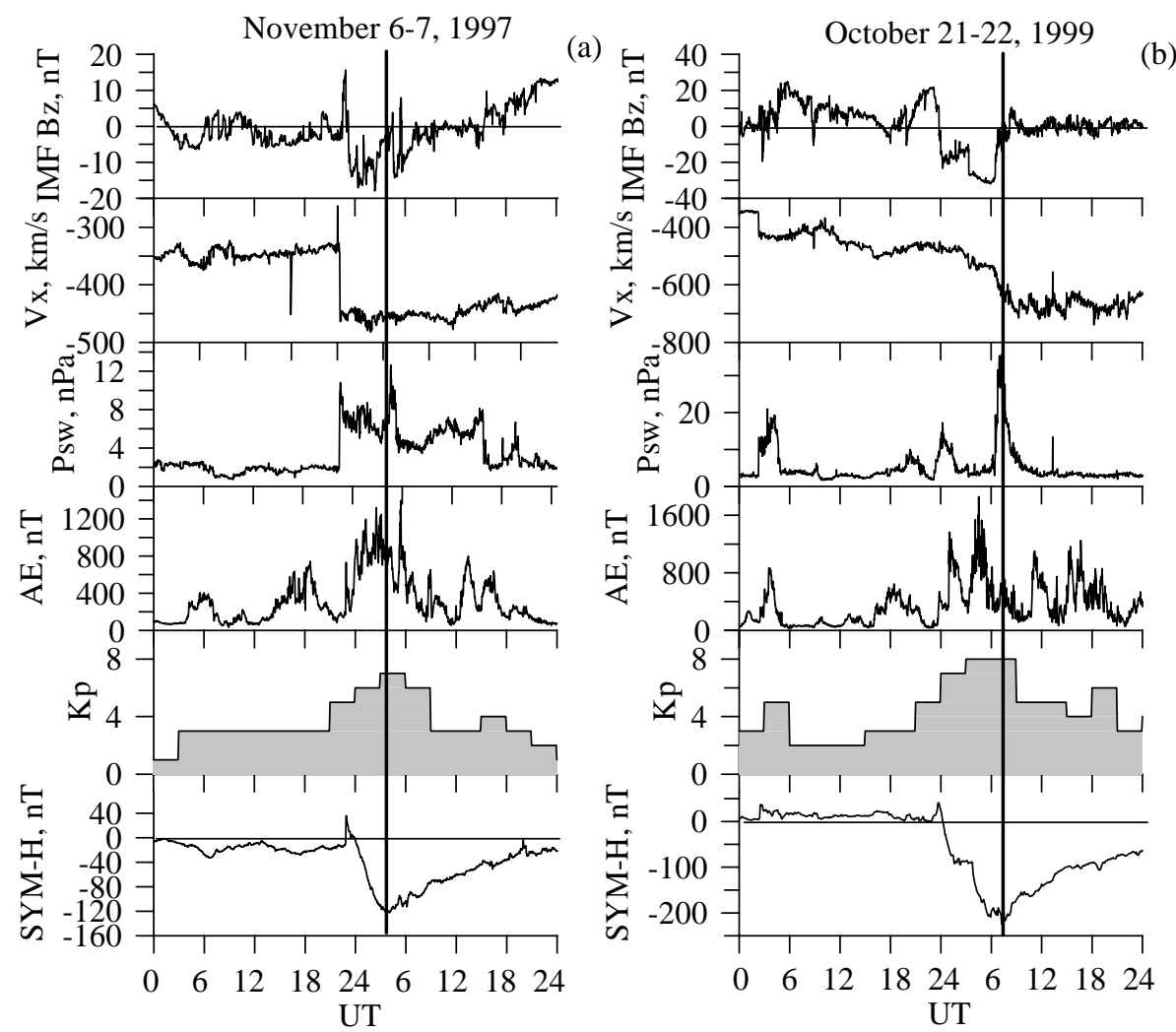

(b)

Fig. 1. Overview of the magnetic storms on (a) 6-7 November 1997 and (b) 21-23 October 1999. The solar wind and IMF data were obtained from Wind spacecraft taking into account the time shift of about $40 \mathrm{~min}$. AE, Kp and SymH indices data were obtained from World Data Center C2 for Geomagnetism, Kyoto. Thick black vertical lines mark the time moment of minimum in SYM-H index through all panels at both figures.

influence the total proton ring current energy content (evolution, peak values and location) during storm times?

2. How does the method of modeled SymH* calculation influence the magnitude and time history of this quantity during storms?

The paper is organized as follows. First, we present two storm events, one moderate (SymH minimum of $-120 \mathrm{nT}$ ) on 6-7 November 1997, and one intense (SymH minimum of $-230 \mathrm{nT}$ ) on 21-22 October 1999, which we have modeled. Section 3 presents our modeling approaches, including the description of the IMPTAM model and methods of model SymH* calculation used in this study. In Sects. 4 and 5 we present modeling results of the ring current energy profiles, peak magnitudes and their UT locations for both storm events. In Sect. 6 we summarize our results and present the conclusions.

\section{Overview of modeled storm events: 6-7 November 1997 and 21-23 October 1999}

Two storm events were selected for the present study. Figure 1 presents an overview of the magnetic storms on 67 November 1997 and 21-23 October 1999. The solar wind and IMF data were obtained from the Wind spacecraft, including about 40 min time shift for propagation to the Earth's magnetopause. The AE, $\mathrm{Kp}$ and SymH indices data were obtained from the World Data Center C2 for Geomagnetism, Kyoto.

A moderate intensity storm occurred on 6-7 November 1997 (Fig. 1a). On 6 November IMF $B_{\mathrm{Z}}$ (first panel) fluctuated around zero and dropped to $-15 \mathrm{nT}$ at the end of the day around 23:00 UT. Together with the $B_{\mathrm{Z}}$ drop, at 22:10 UT there was an increase of the $V_{\mathrm{x}}$ component of the solar wind speed (second panel) from $330 \mathrm{~km} \mathrm{~s}^{-1}$ up to $460 \mathrm{~km} \mathrm{~s}^{-1}$. On 6 November, the solar wind dynamic pressure (third panel) was about $3 \mathrm{nPa}$, increasing up to about $10 \mathrm{nPa}$ at about 22:00 UT. The AE index (fourth panel) had several peaks with its highest magnitudes of about $1300 \mathrm{nT}$ at 02:00 UT and 1400 nT at 05:00 UT on of 7 November. The $\mathrm{Kp}$ index (fifth panel) reached 7 during the storm maximum. 
The SymH index (sixth panel) reached $-120 \mathrm{nT}$ at about 04:00 UT on 7 November and recovered to $-20 \mathrm{nT}$ by the end of the day.

Figure $1 \mathrm{~b}$ shows an overview of the intense storm on 2123 October 1999. IMF $B_{\mathrm{Z}}$ (first panel) turned from $+20 \mathrm{nT}$ to $-20 \mathrm{nT}$ at about 23:50 UT on 21 October and after some increase during the next three hours dropped to $-30 \mathrm{nT}$ around 06:00 UT on 22 October. After that, the IMF $B_{\mathrm{Z}}$ oscillated around zero. The $V_{\mathrm{x}}$ component of solar wind velocity (second panel) increased rather gradually from $480 \mathrm{~km} \mathrm{~s}^{-1}$ at 20:10 UT on 21 October up to $700 \mathrm{~km} \mathrm{~s}^{-1}$ at $02: 58 \mathrm{UT}$ on 22 October. Solar wind dynamic pressure (third panel) showed two main peaks, a $15 \mathrm{nPa}$ peak around 24:00 UT on 21 October and a $35 \mathrm{nPa}$ peak around 07:00 UT on 22 October. There were several peaks in the AE index (fourth panel) reaching 800-1600 nT. The Kp index (fifth panel) increased to 5 from 03:00 to 06:00 UT on 21 October, and reached 8 during the storm maximum around 06:00 UT on 22 October. The SymH index (sixth panel) dropped to $-230 \mathrm{nT}$ at 06:0007:00 UT on 22 October. Thick black vertical lines mark the time moment of minimum in the SymH index in both figures.

\section{Modeling approach}

\subsection{Inner magnetosphere particle transport and accel- eration model}

The inner magnetosphere particle transport and acceleration model (IMPTAM), developed by Ganushkina et al. (2001, 2005, 2006), follows distributions of ions and electrons with arbitrary pitch angles from the plasma sheet to the inner Lshell regions with energies reaching up to hundreds of keVs in time-dependent magnetic and electric fields. We trace a distribution of particles in the guiding center, or drift, approximation, in which we can picture the motion of a charged particle as displacements of its guiding center, or the center of the circular Larmor orbit of a moving particle. The guiding center theory assumes that the electromagnetic fields are known and can be used in geophysical plasmas, where the external field is strong and will not be changed much by the motion of the particle themselves.

As guiding center drifts we take into account $\boldsymbol{E} \times \boldsymbol{B}$ drift, where $\boldsymbol{E}$ and $\boldsymbol{B}$ are electric and magnetic fields, respectively, and magnetic drift, which, in its turn, includes gradient and curvature drifts. The drift velocity is a combination of the velocity $V_{E \times B}$ due to $\boldsymbol{E} \times \boldsymbol{B}$ drift $V_{E \times B}=(\boldsymbol{E} \times \boldsymbol{B}) / B^{2}$ and the velocities of gradient $V_{\nabla}$ and curvature $V_{\text {cur }}$ drifts $V_{\nabla}+$ $V_{\text {cur }}=\left(m v_{\perp}^{2}\right) /\left(2 q B^{2}\right)(\boldsymbol{B} \times \nabla B)+\left(m v_{\|}^{2}\right) /\left(q R_{\mathrm{c}}^{2} B^{2}\right)\left(\boldsymbol{R}_{\mathrm{c}} \times B\right)$ (Roederer, 1970), where $m$ is the particle mass, $q$ is the particle charge, $v_{\perp}$ and $v_{\|}$are the particle velocities perpendicular and parallel to the magnetic field, respectively, $R_{\mathrm{c}}$ is the radius of curvature of magnetic field line $\left(\nabla_{\perp} B=\right.$ $-\left(B / R_{\mathrm{C}}\right) \boldsymbol{n}$, where $\boldsymbol{n}$ is the unit normal vector along the radius of curvature).
We assume that the first and second adiabatic invariants are conserved. The first adiabatic invariant for nonrelativistic particles is the particle magnetic moment given by $\mu=p_{\perp}^{2} /(2 m B)$, where $p_{\perp}=p$ is the particle's momentum in the guiding center system (Roederer, 1970). The magnetic moment of a particle is conserved in all cases, even in nonstationary fields, as long as the guiding center approximation is valid. There are two main conditions for guiding center approximation:

1. Spatial variations in the area of Larmor radius $\rho_{\mathrm{c}}$ are very small, so that $\rho_{\mathrm{c}}\left(\nabla_{\perp} B / B\right) \ll 1$. Magnetic field $\boldsymbol{B}$ does not vary much along the Larmor orbit.

2. Temporal variations are small in comparison with Larmor period $\tau_{\mathrm{c}}\left(\nabla_{\perp} B / B\right) \ll 1$.

Second adiabatic invariant is related to the bounce motion of a particle along magnetic field line. The integral $J=\oint p_{\|} d s$, taken along a given fixed magnetic field line for a complete bounce cycle, where $p_{\|}$is the particle's momentum parallel to the magnetic field, and $d s$ is the length element of a field line, is an adiabatic invariant and is conserved during the drift of a trapped particle as long as field variations during the time of a bounce period $\tau_{\mathrm{b}}$ are small $\left(\tau_{\mathrm{b}} d B / d t\right) / B \ll 1$. The particle's momentum $p$ is constant during one bounce, so $J=2 p I$, where $I=\int_{S_{\mathrm{m}}}^{S_{\mathrm{m}}^{\prime}}\left[1-B(s) / B_{\mathrm{m}}\right]^{1 / 2} d s, S_{\mathrm{m}}$ and $S_{\mathrm{m}}^{\prime}$ are the mirror points, $B(s)$ is the magnetic field along magnetic field line, $B_{\mathrm{m}}$ is the magnetic field at the mirror point.

With the above mentioned assumptions, we consider bounce-average drift velocity after averaging over one bounce of $\boldsymbol{E} \times \boldsymbol{B}$ magnetic drift velocities (Roederer, 1970)

$\left\langle v_{0}\right\rangle=\frac{\boldsymbol{E}_{0} \times \boldsymbol{B}_{0}}{B_{0}^{2}}+\frac{2 p}{q \tau_{\mathrm{b}} B_{0}} \nabla I \times \boldsymbol{e}_{0}$,

where $\boldsymbol{E}_{0}$ and $\boldsymbol{B}_{0}$ are the electric and magnetic fields in the equatorial plane, respectively, $\boldsymbol{e}_{0}$ is the unit vector in the direction of the magnetic field $\boldsymbol{B}_{0}$.

In order to follow the evolution of the particle distribution function $f$ and particle fluxes in the inner magnetosphere dependent on the position $\boldsymbol{R}$, time $t$, energy $E_{\text {kin }}$, and pitch angle $\alpha$, it is necessary to specify:

1. particle distribution at initial time at the model boundary;

2. magnetic and electric fields everywhere dependent on time;

3. drift velocities;

4. all sources and losses of particles.

Generally, the changes in the distribution function $f\left(R, \phi, t, E_{\mathrm{kin}}, \alpha\right)$, where $R$ and $\phi$ are the radial and azimuthal coordinates in the equatorial plane, respectively, $t$ 
is the time, $E_{\text {kin }}$ is the particle energy, $\alpha$ is the particle pitch angle, are obtained by solving the following equation:

$\frac{d f}{d t}=\frac{\partial f}{\partial \phi} \cdot V_{\phi}+\frac{\partial f}{\partial R} \cdot V_{R}+$ sources - losses,

where $V_{\phi}$ and $V_{R}$ are the azimuthal and radial components of the bounce-average drift velocity.

At the beginning of modeling with IMPTAM, the inner magnetosphere is considered empty. In this case, only the effects of newly entering particles from the plasma sheet are investigated. Ganushkina et al. (2006) have studied the effects of the initial distribution on the storm-time ring current formation. For the 21-25 April 2001 storm, the modeling with an initially filled inner magnetosphere resulted in an increase by a factor of about 1.7 of the peak magnitude of the total proton ring current energy. The preexisting particles are subject to loss quickly after the newly injected particles occupy the inner magnetosphere.

The model boundary is set in the plasma sheet at distances, depending on the scientific questions we are trying to answer, from $6.6 R_{\mathrm{E}}$ to $10 R_{\mathrm{E}}$. The particle distribution at the boundary is defined as a Maxwellian or kappa distribution function with parameters obtained from the empirical relations or from the observations during specific events.

Liouville's theorem states that, in the absence of external forces and losses, the distribution function remains constant along the dynamic trajectory of particles. This theorem is used to gain information of the entire distribution function. If we know the distribution function $f\left(R, \phi, t, E_{\mathrm{kin}}, \alpha\right)$ of particles at a time moment $t_{1}$, then we can obtain the distribution function of particles at a time moment $t_{2}=t_{1}+\Delta t$, by computing the drift velocity of the particles. The distribution function at $t_{2}$ will not be the same as at $t_{1}$ at the corresponding positions, since we need to take into account the phasespace-dependent losses ( $\left.\tau_{\text {loss }}\right)$. The final distribution function at $t_{2}$ will be $f\left(t_{2}\right)=f\left(t_{1}\right) \exp \left(-\Delta t / \tau_{\text {loss }}\right)$.

Particle loss processes, which are important for modeling the ring current ions, include charge-exchange with neutral hydrogen in the upper atmosphere, Coulomb collisions, and convective outflow through the magnetopause. The chargeexchange cross-section is obtained from Janev and Smith (1993). The thermosphere model MSISE 90 (Hedin, 1991) and the plasmasphere model by Carpenter and Anderson (1992) are used.

An advantage of the IMPTAM is that it can simulate the full pitch-angle distribution of particles and utilize any magnetic or electric field configurations (see below the list of representations used in the present study). In addition to the large-scale fields, transient fields associated with the dipolarization process in the magnetotail during substorm onset are included as an earthward propagating electromagnetic pulse of localized radial and longitudinal extent (Li et al., 1998; Sarris et al., 2002). The magnetic field disturbance from this dipolarization process was obtained from Faraday's law. One of the important results obtained from IMPTAM modeling is the ability to simulate several pulses launched at successive substorm onset times (Ganushkina et al., 2001, 2005, 2006) to reproduce the observed amount of ring current protons with energies $>80 \mathrm{keV}$ during a storm recovery phase (Ganushkina et al., 2006), which could not be achieved using other models for the magnetic field and large-scale convection electric field.

IMPTAM has also been used (Ganushkina et al., 2004, 2005,2006 ) to examine the evolution of the current systems during magnetic storms, to compute energetic ion drifts in the inner magnetosphere, and to evaluate the magnetospheric sources of magnetic disturbances recorded on ground (i.e., the sources of the Dst index).

\subsection{Representations for magnetic and electric fields and boundary conditions used in simulations}

The evolution of modeled proton distributions was followed using combinations of several representations for magnetic and electric fields with different boundary conditions. We use a dipole for the internal magnetic field. For the external magnetic field four different representations were used:

1. no external field sources (dipole only);

2. Kp-dependent Tsyganenko T89c (T89 abbreviation is used throughout the paper) (Tsyganenko, 1989; Peredo et al., 1993);

3. T96 (Tsyganenko, 1995) with Dst, $P_{\mathrm{sw}}, \mathrm{IMF} B_{\mathrm{y}}$ and $B_{\mathrm{z}}$ as input parameters;

4. Tsyganenko and Sitnov TS04 (Tsyganenko and Sitnov, 2005) with Dst, $P_{\mathrm{sw}}, \mathrm{IMF} B_{\mathrm{y}}$ and $B_{\mathrm{z}}$ and six variables $W_{i}, i=1,6$ as input parameters. The variables $W$ enter in the six magnitude coefficients for the magnetic fields from each source and calculated as time integrals dependent on solar wind and IMF parameters from the moment in time when IMF $B_{\mathrm{Z}}$ turns southward.

The electric field representations include

1. Kp-dependent Volland-Stern VS (Volland, 1973; Stern, 1975) convection electric field;

2. Boyle et al. (1997) polar cap potential dependent on solar wind and IMF parameters applied to Volland-Stern type convection electric field field.

Four types of boundary proton distributions used in the model are the following:

1. $\mathrm{BC} 1$ : Maxwellian distribution function at $6.6 R_{\mathrm{E}}$ with $n=0.5 \mathrm{~cm}^{-3}$ and $T=5 \mathrm{keV}$;

2. BC2: Kappa-type distribution function $(\kappa=5)$ with the observed parameters $T(<T>=7.8 \mathrm{keV})$ and $n(<$ $n>=0.97 \mathrm{~cm}^{-3}$ ) by LANL MPA (Bame et al., 1993) in the $3-45 \mathrm{keV}$ energy range at $6.6 R_{\mathrm{E}}$ on the nightside 
November 6-7, 1997, IMPTAM model

\begin{tabular}{|c|c|c|}
\hline $\begin{array}{l}\text { B field } \\
- \text { dipole } \\
- \text { T89 } \\
\text { T } \\
\text { T96 } \\
\text { TS04 }\end{array}$ & \begin{tabular}{c}
\multicolumn{1}{c}{ E field } \\
VS: Volland-Stern \\
Boyle: Boyle et \\
al. (1997)
\end{tabular} & $\begin{array}{l}\text { Boundary conditions } \\
\text { BC1: Maxwell at } 6.6 \mathrm{Re}, \mathrm{n}=0.5 \mathrm{~cm}^{-3}, \mathrm{~T}=5 \mathrm{keV} \\
\text { BC2: kappa at } 6.6 \mathrm{Re}, \mathrm{n}, \mathrm{T}_{\|} \text {and } \mathrm{T}_{\perp} \text { from LANL } \\
\text { BC3: Maxwell at 10 Re, } \mathrm{T}=5 \mathrm{keV}, \mathrm{n}_{\mathrm{ps}}=0.025 \mathrm{n}_{\mathrm{Sw}}+0.395 \\
\text { BC4: Maxwell at 10 Re, T, } \mathrm{n} \text { from Tsyganenko and } \\
\text { Mukai (2003) }\end{array}$ \\
\hline
\end{tabular}
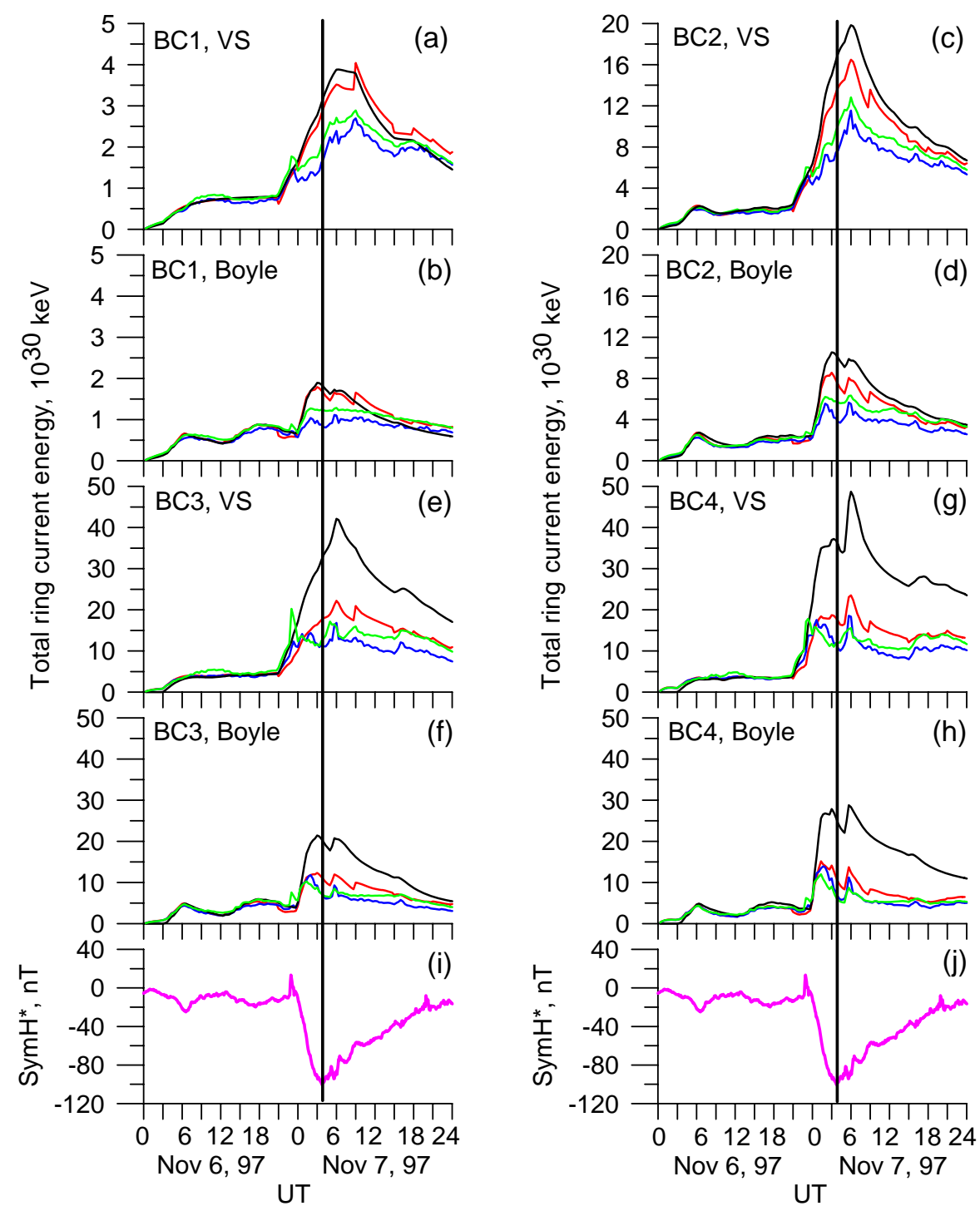

Fig. 2. Modeled proton (1-300 keV) ring current energy content during the 6-7 November 1997 storm with initially empty magnetosphere for combinations of four boundary conditions, two electric and four magnetic field representations. The corrected SymH* index is shown by thick purple lines. Thick black vertical lines mark the time moment of minimum in SymH* index through all figures. Note the scale differences on the panels.

part of the boundary (18:00-06:00 MLT) in the equatorial plane;

3. BC3: Maxwellian distribution function at $10 R_{\mathrm{E}}$ with parameter $n$ given by the empirical relation between the plasma sheet number density and the solar wind num- ber density $n_{\mathrm{ps}}=0.025 n_{\mathrm{sw}}+0.395$ (Ebihara and Ejiri, 2000) and $T=5 \mathrm{keV}$;

4. BC4: Maxwellian distribution function at $10 R_{\mathrm{E}}$ with $n$ and $T$ given by the empirical model derived from Geotail data by Tsyganenko and Mukai (2003). 
October 21-23, 1999, IMPTAM model

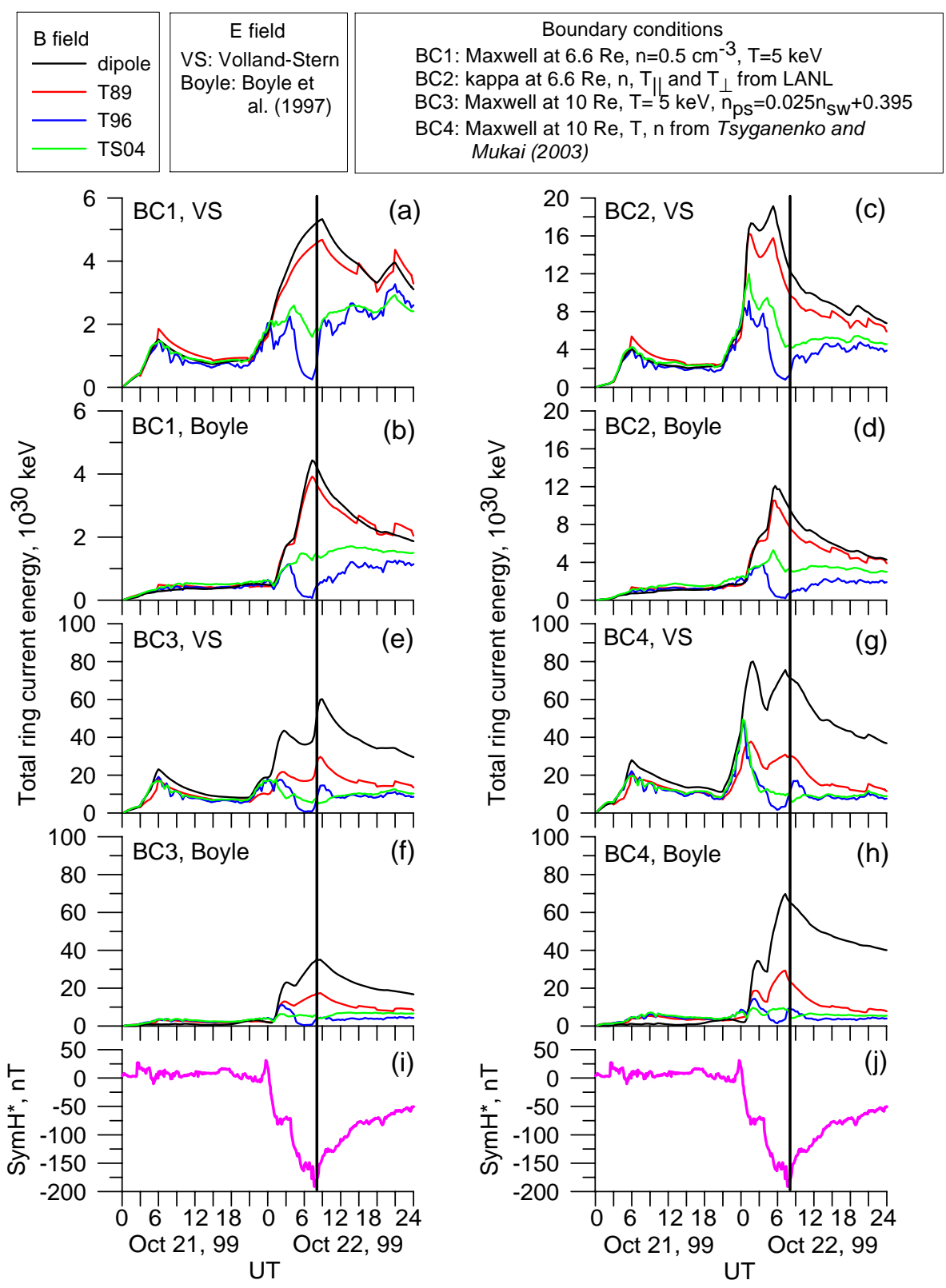

Fig. 3. Similar to Fig. 2 but for intense storm on 21-22 October 1999.

\subsection{Methods of calculation of model Dst index}

The corrected Dst* includes the pressure correction (Burton et al., 1975) and the contribution from induced currents

Dst $^{*}=\frac{\text { Dst }-7.26 \sqrt{P_{\mathrm{sw}}}+11.0}{1.3}$

with removed influence of the magnetopause currents $\left(7.26 \sqrt{P_{\mathrm{sw}}}\right)$, a correction factor for the contribution from induced currents within the Earth (1.3) (Häkkinen et al., 2002) and a quiet-time offset value (11.0 nT) also taken into account. We assume that the remaining contents of Dst* mainly consist of the contributions from the ring and the tail currents. We use the corrected $1 \mathrm{~min} \mathrm{SymH}^{*}$ index for our comparisons, instead of the $1 \mathrm{~h}$ Dst* index, to have better time resolution.

Two different methods are used for our calculations of the modeled SymH* time series. One of them is the widely used Dessler-Parker-Sckopke relation DPS (Dessler and Parker, 1959; Sckopke, 1966)

$\mathrm{SymH}_{\mathrm{DPS}}^{*}[\mathrm{nT}]=-3.98 \times 10^{-30} \mathrm{E}_{\mathrm{RC}}[\mathrm{keV}]$, 
November 6-7, 1997, IMPTAM model

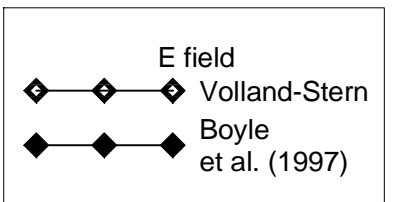

Boundary conditions

BC1: Maxwell at $6.6 \mathrm{Re}, \mathrm{n}=0.5 \mathrm{~cm}^{-3}, \mathrm{~T}=5 \mathrm{keV}$

BC2: kappa at $6.6 \mathrm{Re}, \mathrm{n}, \mathrm{T}_{\|}$and $\mathrm{T}_{\perp}$ from $\mathrm{LANL}$

BC3: Maxwell at $10 \mathrm{Re}, \mathrm{T}=5 \mathrm{keV}, \mathrm{n}_{\mathrm{ps}}=0.025 \mathrm{n}_{\mathrm{sw}}+0.395$

BC4: Maxwell at $10 \mathrm{Re}, \mathrm{T}, \mathrm{n}$ from Tsyganenko and Mukai (2003)
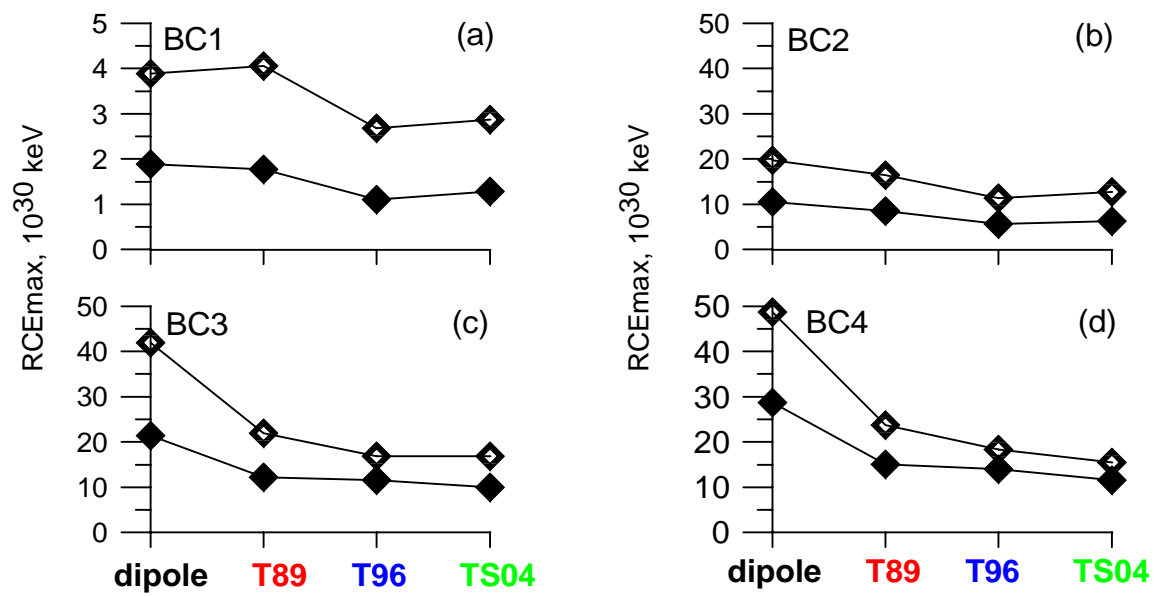

Fig. 4. Peak magnitudes of the ring current energy profiles shown in Fig. 2 during 6-7 November 1997 storm.

which relates the total energy content $E_{\mathrm{RC}}$ of the plasma within the inner magnetosphere to a magnetic perturbation at the center of the Earth $\mathrm{SymH}_{\mathrm{DPS}}^{*}$.

Another method uses the Biot-Savart law to derive the magnetic disturbance induced by the current. The current density $\boldsymbol{J}_{\perp}$ perpendicular to the magnetic field $\boldsymbol{B}$ is given by

$\boldsymbol{J}_{\perp}=\frac{\boldsymbol{B}}{B^{2}} \times\left[\nabla P_{\perp}+\left(P_{\|}-P_{\perp}\right) \frac{(\boldsymbol{B} \cdot \nabla) \boldsymbol{B}}{B^{2}}\right]$,

where $P_{\|}$and $P_{\perp}$ are plasma pressure, parallel and perpendicular to the magnetic field. This equation is valid if a quasistatic equilibrium exists (force balanced state) and there is no time dependence on the timescale of interest and inertial terms can be neglected. The magnetic disturbance parallel to the Earth's dipole at the center of the Earth $\triangle B$ is induced by the azimuthal component $J_{\phi}$ of $\boldsymbol{J}_{\perp}$

$\triangle B=\frac{\mu_{0}}{4 \pi} \int_{\mathrm{r}} \int_{\lambda} \int_{\phi} \cos ^{2} \lambda J_{\phi}(r, \lambda, \phi) d r d \lambda d \phi$,

where $\mu_{0}$ is the magnetic permeability, $r$ is the radial distance, $\lambda$ is the latitude, $\phi$ is the MLT and $J_{\phi}$ is given by

$$
\begin{aligned}
J_{\phi}= & \frac{1}{B^{2}}\left(\frac{B_{\mathrm{r}}}{r} \frac{\partial P_{\perp}}{\partial \lambda}-B_{\lambda} \frac{\partial P_{\perp}}{\partial r}\right) \\
& +\frac{1}{B^{3}}\left(P_{\|}-P_{\perp}\right)\left(\frac{B_{\mathrm{r}}}{r} \frac{\partial B}{\partial \lambda}-B_{\lambda} \frac{\partial B}{\partial r}\right)
\end{aligned}
$$

\section{Modeling results: ring current energy content}

\subsection{Ring current energy profiles}

Figure 2 shows the evolution of modeled proton ring current energy in units of $10^{30} \mathrm{keV}$ during the 6-7 November 1997 storm for several combinations of the magnetic and electric fields and boundary conditions. At each panel $(\mathrm{a}-\mathrm{h})$ the ring current energy computed using different magnetic fields is shown by different colors: dipole (thick black lines), T89 (red lines), T96 (blue lines), and TS04 (green lines). The corrected SymH* index (i, j) with pressure correction and with quiet-time offset and removed contribution from induced currents within the Earth is shown by thick purple lines. Thick black vertical lines mark the time of minimum SymH* through all figures. Note that the scales are different for panels (a), (b), (c), (d) and (e)-(h), but they are the same for the same boundary conditions but different electric fields ( $\mathrm{a}$ and $\mathrm{b}$ for $\mathrm{BC} 1$ ), ( $\mathrm{c}$ and $\mathrm{d}$ for $\mathrm{BC} 2$ ) and for all combinations for $\mathrm{BC} 3$ and $\mathrm{BC} 4$.

Figure 3 shows, similarly to Fig. 2, the evolution of modeled proton ring current energy in $10^{30} \mathrm{keV}$ during the 21 22 October 1999 intense storm for several combinations of magnetic and electric fields and boundary conditions.

Table 1 summarizes the results on storm-time ring current energy profiles for both storm events, moderate on 67 November 1997 and intense on 21-22 October 1999.

and $\triangle B$ is an estimate of the corrected $\mathrm{SymH}^{*}$ index. 
Table 1. Model-dependent storm-time ring current: ring current energy profiles.

\begin{tabular}{|c|c|}
\hline Moderate storm & Intense storm \\
\hline \multicolumn{2}{|c|}{ Before storm: filling of initially empty magnetosphere } \\
\hline $\begin{array}{l}\text { - all magnetic field representations produce close val } \\
\text { - following SymH* profile in opposite with Boyle } \\
\text { - magnitudes of } 0.5-20 \times 10^{30} \mathrm{keV}\end{array}$ & $\begin{array}{l}\text { les for given boundary conditions } \\
\text { - peak at 06:00 UT on } 21 \text { Oct with VS } \\
\text { - magnitudes of } 1-5 \times 10^{30} \mathrm{keV}\end{array}$ \\
\hline \multicolumn{2}{|c|}{ Storm beginning: sharp increase in ring current energy } \\
\hline \multicolumn{2}{|c|}{$\begin{array}{l}\text { - no dependence on magnetic field } \\
\text { - coincides well with SymH* decrease for Boyle field, } 3 \mathrm{~h} \text { earlier for VS field }\end{array}$} \\
\hline \multicolumn{2}{|c|}{ Storm maximum and early recovery } \\
\hline $\begin{array}{l}\text { - vary significantly (shape, magnitudes, peak(s) locat } \\
\text { parameters } \\
\text { - } 2 \text { times smaller values with Boyle than with VS for } \\
\text { - largest values with dipole, then T89, then TS04 an } \\
\text { - observed SymH* not followed by any combina- } \\
\text { tion of fields and boundary conditions } \\
\text { - magnitudes of } 4-45 \times 10^{30} \mathrm{keV}\end{array}$ & $\begin{array}{l}\text { ons) dependent on the background fields and their } \\
\text { all magnetic fields and boundary conditions } \\
\text { T96 with complex profiles with multiple peaks } \\
\text { - two drops in observed SymH* followed by dipole } \\
\text { and T89 and VS and Boyle and for BC2, BC3, BC4 } \\
\text { - magnitudes of } 4-80 \times 10^{30} \mathrm{keV}\end{array}$ \\
\hline
\end{tabular}

Boundary conditions at $6.6 R_{\mathrm{E}}$

- LANL time-dependent BCs give 5 times increase compared to stationary BCs

Boundary conditions at $10 R_{\mathrm{E}}$

-1.5 times increase vs. time-varying $\mathrm{BC}$ at $6.6 R_{\mathrm{E}} \mid-2$ times increase vs. time-dependent $\mathrm{BC}$ at $6.6 R_{\mathrm{E}}$

- significantly larger values with dipole for VS and Boyle fields

\subsection{Peak magnitudes of the ring current energy and their UTs}

In order to better examine and highlight the similarities and differences between the model configurations within IMPTAM, the results were distilled to only the peak magnitudes of the ring current intensity. Figure 4 presents these peak magnitudes in units of $10^{30} \mathrm{keV}$ of the ring current energy profiles shown in Fig. 2 during the 6-7 November 1997 storm for four boundary conditions (BC1-BC4) for the VollandStern (open diamonds) and the Boyle (black diamonds) electric fields as dependent on four magnetic fields (dipole, T89, T96, and TS04). Peak magnitudes of the ring current energy profiles for the intense 21-22 October 1999 storm are shown in Fig. 5 similarly to those for the moderate storm on 6-7 November 1997 (Fig. 4).

Another distillation of the time series plots in Figs. 2 and 3 is to consider the offset in Universal Time (UT) between the peak of the modeled ring current energy content and the $\mathrm{SymH}^{*}$ minimum. Figure 6 presents the times of the peak magnitudes of the ring current energy profiles shown in Fig. 2 during the 6-7 November 1997 storm in terms of the difference $\Delta \mathrm{UT}_{\mathrm{RCE}}=\mathrm{UT}\left(\mathrm{RCE}_{\max }\right)-\mathrm{UT}\left(\mathrm{SymH}_{\min }\right)$ between $\mathrm{UT}\left(\mathrm{RCE}_{\max }\right)$, when the maximum of the ring current energy is reached during the storm and $\mathrm{UT}\left(\mathrm{SymH}_{\min }\right)$, when the $\mathrm{SymH}^{*}$ index dropped to its minimum. Negative values of $\Delta \mathrm{UT}_{\mathrm{RCE}}$ mean before and positives values mean after the storm maximum as defined by the UT of the SymH* minimum, respectively. It is worth mentioning that the locations of peak values of the ring current energy can be determined rather easily for the dipole and T89 magnetic field, whereas it is more difficult to do when using the T96 and TS04. The ring current energy profiles for the T96 and TS04 exhibit more variations and peaks. In the present study in Figs. 4 and 6 the largest peaks are plotted.

Locations of the peak magnitudes of the ring current energy profiles during the intense 21-22 October 1999 storm are presented in Fig. 7 similarly to Fig. 6.

Table 2 contains the main features of the storm-time ring current energy peak values and their UTs for both storm events, moderate on 6-7 November 1997 and intense on 2122 October 1999.

\section{Modeling results: global magnetic field depression}

\subsection{Model SymH* profiles computed by Biot-Savart and Dessler-Parker-Sckopker DPS relation methods}

The focus of this part of the study centers on the method of converting the ring current energy content into a global 
October 21-23, 1999, IMPTAM model
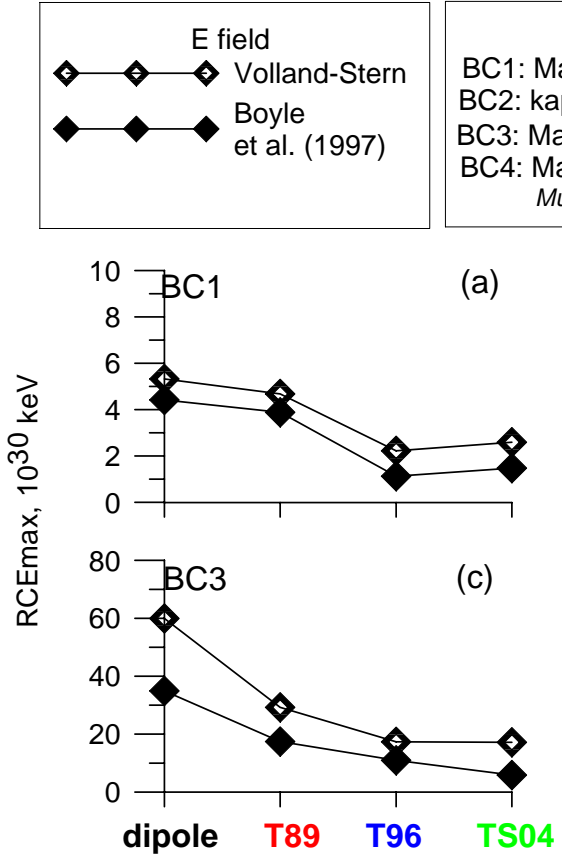

Boundary conditions

BC1: Maxwell at $6.6 \mathrm{Re}, \mathrm{n}=0.5 \mathrm{~cm}^{-3}, \mathrm{~T}=5 \mathrm{keV}$

BC2: kappa at $6.6 \mathrm{Re}, \mathrm{n}, \mathrm{T}_{\|}$and $\mathrm{T}_{\perp}$ from $\mathrm{LANL}$

BC3: Maxwell at $10 \mathrm{Re}, \mathrm{T}=5 \mathrm{keV}, \mathrm{n}_{\mathrm{ps}}=0.025 \mathrm{n}_{\mathrm{sw}}+0.395$

BC4: Maxwell at $10 \mathrm{Re}, \mathrm{T}, \mathrm{n}$ from Tsyganenko and Mukai (2003)

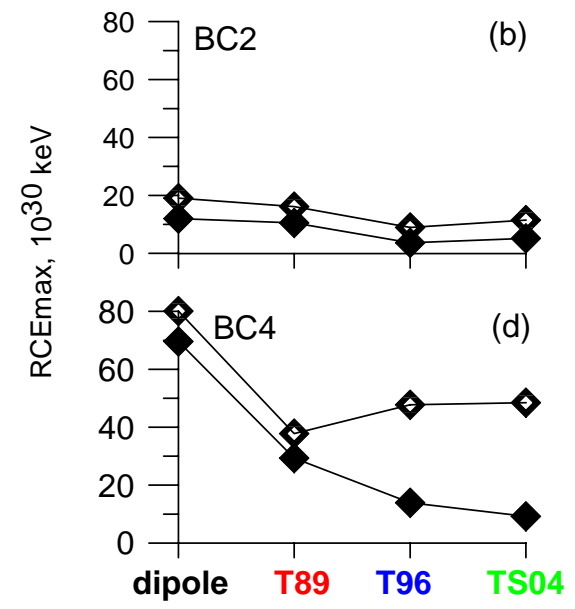

Fig. 5. Peak magnitudes of the ring current energy profiles shown in Fig. 3 during 21-22 October 1999 storm, similar to Fig. 4.

November 6-7, 1997, IMPTAM model

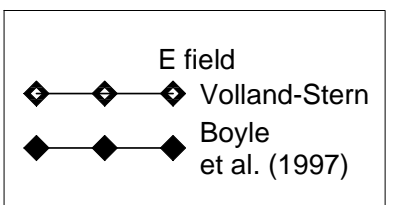

\section{Boundary conditions}

BC1: Maxwell at $6.6 \mathrm{Re}, \mathrm{n}=0.5 \mathrm{~cm}^{-3}, \mathrm{~T}=5 \mathrm{keV}$

BC2: kappa at $6.6 \mathrm{Re}, \mathrm{n}, \mathrm{T}_{\|}$and $\mathrm{T}_{\perp}$ from LANL

BC3: Maxwell at $10 \mathrm{Re}, \mathrm{T}=5 \mathrm{keV}, \mathrm{n}_{\mathrm{ps}}=0.025 \mathrm{n}_{\mathrm{sw}}+0.395$

BC4: Maxwell at $10 \mathrm{Re}, \mathrm{T}, \mathrm{n}$ from Tsyganenko and Mukai (2003)
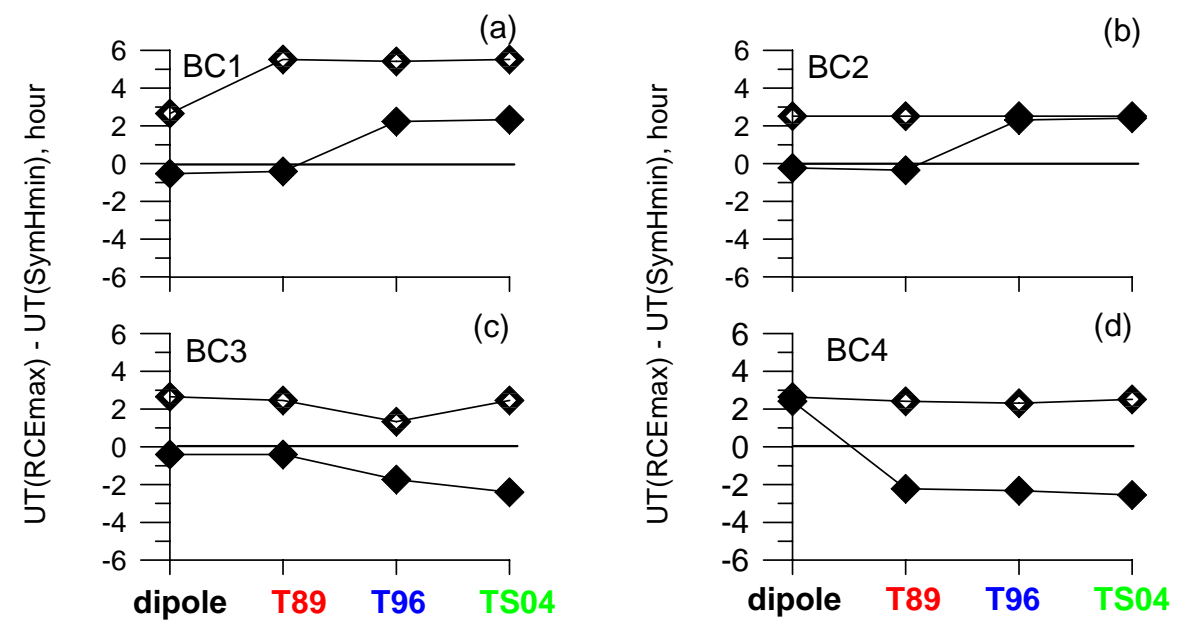

Fig. 6. Times of the peak magnitudes of the ring current energy profiles shown in Fig. 2 during 6-7 November 1997 storm in terms of the difference $\Delta \mathrm{UT}_{\mathrm{RCE}}=\mathrm{UT}\left(\mathrm{RCE}_{\max }\right)-\mathrm{UT}\left(\mathrm{SymH}_{\min }\right)$ (see the text).

magnetic perturbation for direct comparison with SymH*. Figure 8 presents the modeled $\mathrm{SymH}^{*}$ computed as magnetic field depression at the center of the Earth produced by the modeled proton $(1-300 \mathrm{keV})$ distribution during the 6 7 November 1997 storm for boundary conditions BC1 and BC2 set at geostationary orbit $6.6 R_{\mathrm{E}}$. The Volland-Stern 
October 21-23, 1999, IMPTAM model

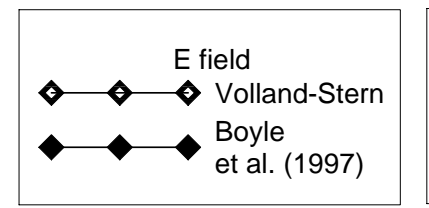

BC1: Maxwell at $6.6 \mathrm{Re}, \mathrm{n}=0.5 \mathrm{~cm}^{-3}, \mathrm{~T}=5 \mathrm{keV}$

BC2: kappa at 6.6 Re, $n, T_{\|}$and $T_{\perp}$ from LANL

BC3: Maxwell at $10 \mathrm{Re}, \mathrm{T}=5 \mathrm{keV}, \mathrm{n}_{\mathrm{ps}}=0.025 \mathrm{n}_{\mathrm{sw}}+0.395$

BC4: Maxwell at $10 \mathrm{Re}, \mathrm{T}, \mathrm{n}$ from Tsyganenko and Mukai (2003)
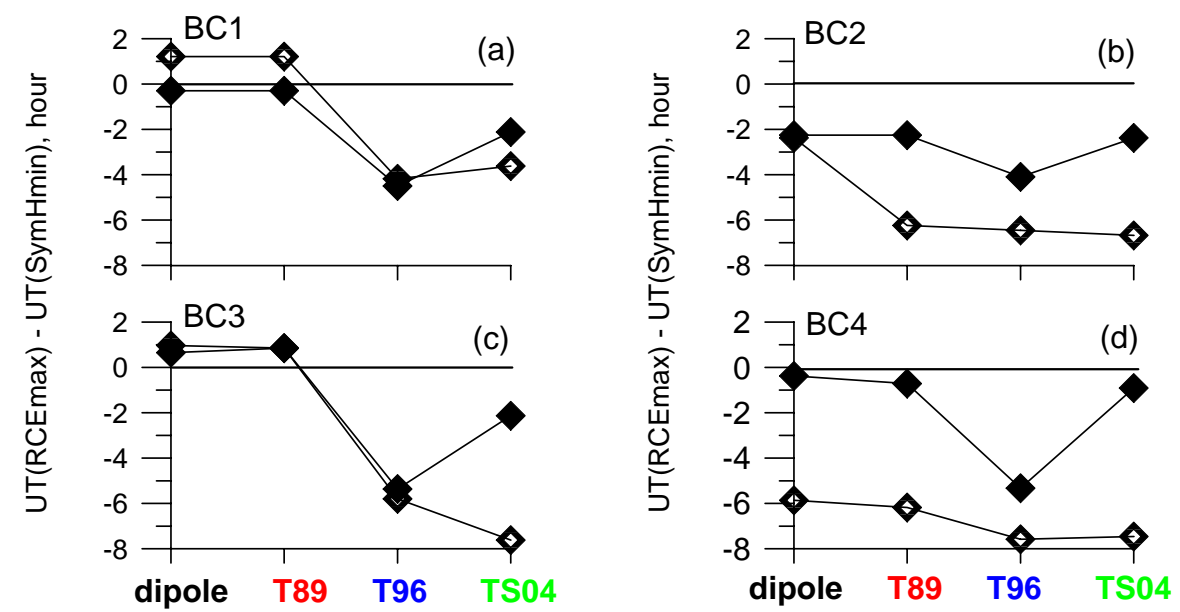

Fig. 7. Times of the peak magnitudes of the ring current energy profiles shown in Fig. 3 during 21-22 October 1999 storm, similar to Fig. 6.

Table 2. Model-dependent storm-time ring current: peak values and their UTs.

\begin{tabular}{|c|c|}
\hline Moderate storm & Intense storm \\
\hline \multicolumn{2}{|c|}{ Peak magnitudes } \\
\hline \multicolumn{2}{|c|}{$\begin{array}{l}\text { - peak magnitudes are, in general, quite similar for both moderate and intense storms } \\
\text { - Boyle results in } 2 \text { times smaller peak values compared to VS for all magnetic fields and BCs } \\
\text { - peak magnitudes decrease when moving from dipole to more realistic magnetic fields } \\
\text { - } 5 \text { times increase from stationary to LANL time- } \mid \begin{array}{l}-5 \text { times increase from stationary to LANL time- } \\
\text { dependent BC at } 6.6 R_{\mathrm{E}}, \text { further } 2 \text { times increase }\end{array} \\
\begin{array}{l}\text { dependent BC at } 6.6 R_{\mathrm{E}} \text {, further } 3 \text { times increase } \\
\text { when moving from } 6.6 \text { to } 10 R_{\mathrm{E}}\end{array} \\
\text { - at } 10 R_{\mathrm{E}} \text { not much difference for T89, T96 and TS04 for VS and Boyle fields }\end{array}$} \\
\hline \multicolumn{2}{|c|}{ UT differences of peak magnitudes and SymH* minima } \\
\hline $\begin{array}{l}\text { - best coincidence for Boyle and dipole and } \mathrm{T} 89 \text {, all } \\
\mathrm{BC} \text {, except for } \mathrm{BC} 4 \text { from Geotail at } 10 R_{\mathrm{E}}\end{array}$ & $\begin{array}{l}-\triangle \mathrm{UT}_{\mathrm{RCE}} \text { close to } 0 \text { for dipole and } \mathrm{T} 89 \text { and Boyle } \\
\text { and for } \mathrm{BC} 1, \mathrm{BC} 3 \text { and } \mathrm{BC} 4 \\
\text { - for } \mathrm{BC} 1 \text { and } \mathrm{BC} 3 \text {, VS results in close to zero val- } \\
\text { ues }\end{array}$ \\
\hline $\begin{array}{l}\text { - plus } 2.5 \mathrm{~h} \text { for all magnetic fields for } \mathrm{VS} \text { for } \mathrm{LANL} \\
\text { time-dependent } \mathrm{BC} \text { at } 6.6 \text { and } 10 R_{\mathrm{E}}(\mathrm{BC} 2, \mathrm{BC} 3 \\
\text { and } \mathrm{BC} 4)\end{array}$ & $\begin{array}{l}\text { - Boyle gives } \Delta \mathrm{UT}_{\mathrm{RCE}} \text { values closer to zero than } \\
\mathrm{VS}\end{array}$ \\
\hline $\begin{array}{l}- \text { at } 6.6 R_{\mathrm{E}} \text { for T96 and TS04, UT difference is }+2 \mathrm{~h} \\
\text { for Boyle, at } 10 R_{\mathrm{E}} \text { is }-2 \mathrm{~h}\end{array}$ & $\begin{array}{l}\text { - for T96 and TS04 and for VS, UT difference is } \\
-6 \mathrm{~h} \text { for } \mathrm{BC} 1-\mathrm{BC} 3\end{array}$ \\
\hline
\end{tabular}

VS and Boyle electric fields were used. Panels on the right (Fig. 8b, d, f, h) were calculated using the Dessler-ParkerSckopke DPS relation between the total ring current energy and the magnetic field depression produced by it at the center of the Earth. At each panel, the modeled SymH* was computed using several magnetic field representations such as dipole (black lines), T89 (red lines), T96 (blue lines), and TS04 (green lines). Panels on the left (Fig. 8a, c, e, g) were computed using the Biot-Savart integration of the azimuthal component of the modeled current perpendicular to the magnetic field. For the calculations of the modeled SymH* on these panels, several magnetic field representations were also 
November 6-7, 1997, IMPTAM model

\begin{tabular}{|l|c|}
\hline \multicolumn{1}{|c|}{ E field } & Boundary conditions \\
VS: Volland-Stern & $\mathrm{BC} 1$ : Maxwell at $6.6 \mathrm{Re}, \mathrm{n}=0.5 \mathrm{~cm}^{-3}, \mathrm{~T}=5 \mathrm{keV}$ \\
Boyle: Boyle et al. (1997) & $\mathrm{BC2:}$ kappa at $6.6 \mathrm{Re}, \mathrm{n}, \mathrm{T}_{\|}$and $\mathrm{T}_{\perp}$ from $\mathrm{LANL}$ \\
\hline
\end{tabular}
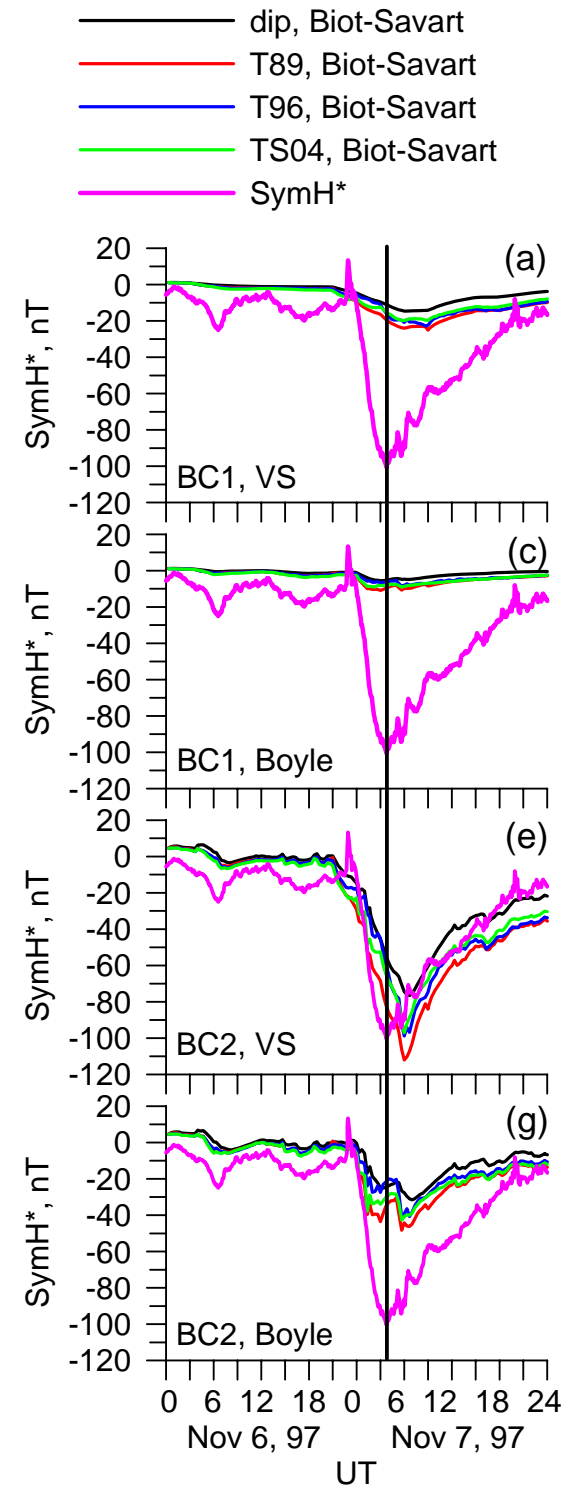

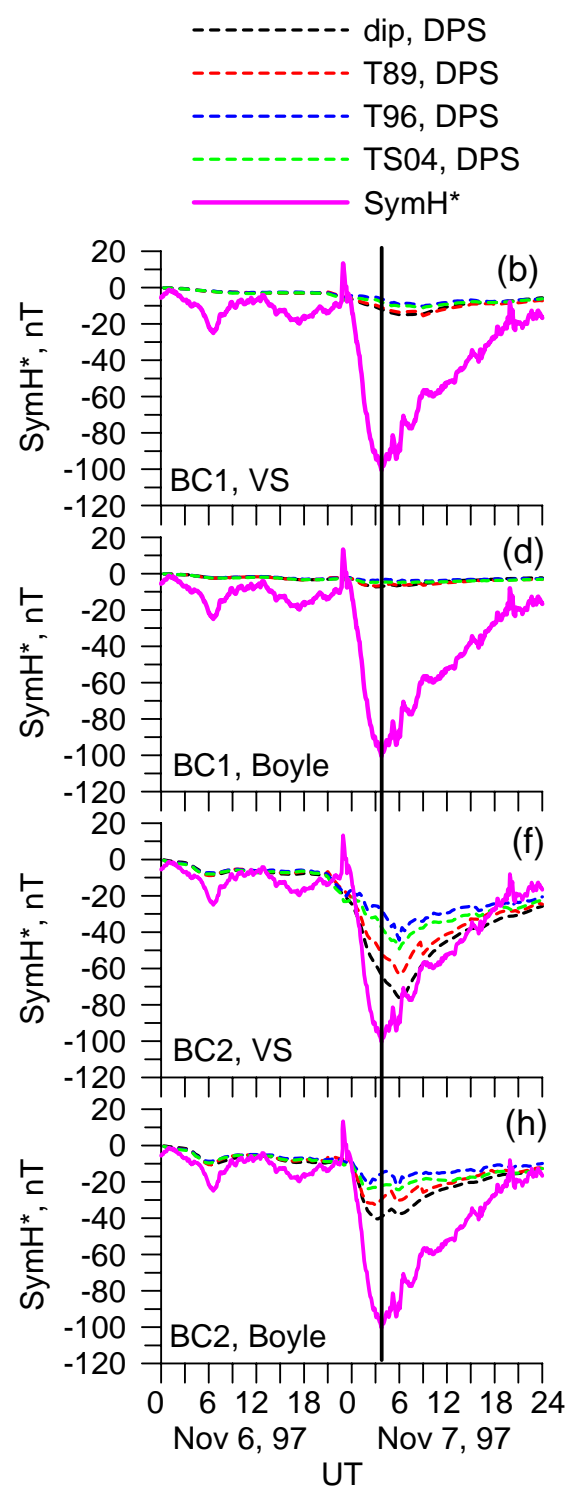

Fig. 8. Magnetic field depression at the center of the Earth produced by the modeled proton (1-300 keV) distribution during 6-7 November 1997 storm with boundary distribution at $6.6 R_{\mathrm{E}}$. Calculations were made by using Biot-Savart's law and Dessler-Parker-Sckopke relation. The observed corrected SymH* index is shown by thick purple lines. Thick black vertical lines mark the time moment of minimum in the observed $\mathrm{SymH}^{*}$ index through all panels.

used such as dipole (dashed black lines), T89 (dashed red lines), T96 (dashed blue lines), and TS04 (dashed green lines). The observed corrected $\mathrm{SymH}^{*}$ index with quiettime offset and removed contribution from induced currents within the Earth is shown by thick purple lines. Thick black vertical lines mark the time moment of the minimum in the observed corrected $\mathrm{SymH}^{*}$ through all panels. Figure 9 presents the modeled SymH*, similarly as Fig. 8 but for boundary conditions $\mathrm{BC} 3$ and $\mathrm{BC} 4$.

Figures 10 and 11 , similarly to Fig. 8 and 9 , respectively, present the modeled $\mathrm{SymH}^{*}$ computed as a magnetic field depression at the center of the Earth produced by the 
November 6-7, 1997 storm, IMPTAM model

\begin{tabular}{|l|c|}
\hline \multicolumn{1}{|c|}{ E field } \\
$\begin{array}{l}\text { VS: Volland-Stern } \\
\text { Boyle: Boyle et al. (1997) }\end{array}$ & $\begin{array}{l}\text { Boundary conditions } \\
\text { BC3: Maxwell at } 10 \mathrm{Re}, \mathrm{T}=5 \mathrm{keV}, \mathrm{n}_{\mathrm{ps}}=0.025 \mathrm{n}_{\mathrm{sw}}+0.395 \\
\text { BC4: Maxwell at 10 Re, T, } \mathrm{n} \text { from Tsyganenko and } \\
\text { Mukai (2003) }\end{array}$ \\
\hline
\end{tabular}
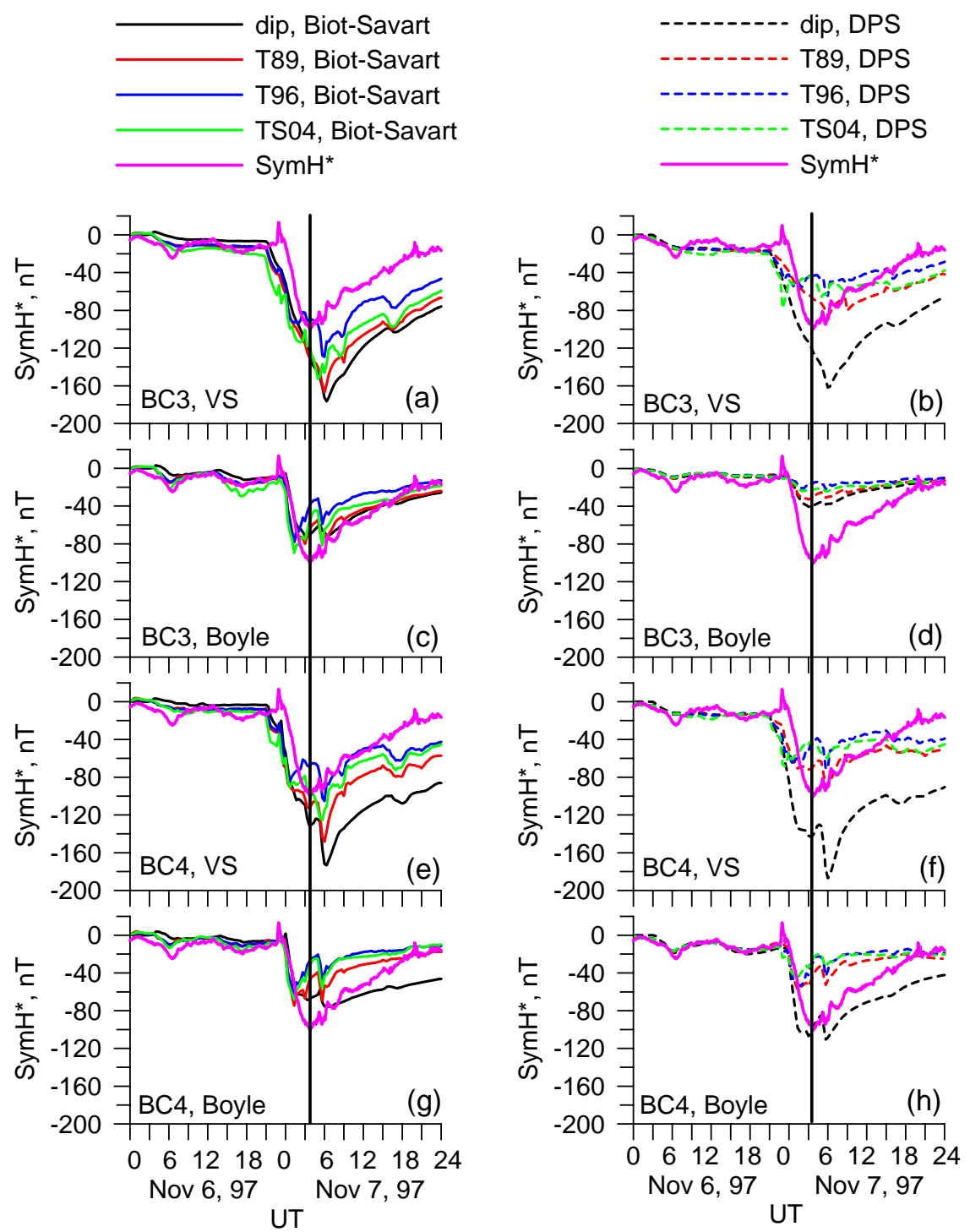

Fig. 9. Similar to Fig. 8 but for boundary distribution at $10 R_{\mathrm{E}}$.

modeled proton (1-300 keV) distribution during the intense 21-22 October 1999 storm for boundary conditions BC1 and $\mathrm{BC} 2$, and $\mathrm{BC} 3$ and $\mathrm{BC} 4$.

Table 3 describes the results on the modeled $\mathrm{SymH}^{*}$ computed using the Dessler-Parker-Sckopke DPS relation and Biot-Savart integration for both storm events, moderate on 6-7 November 1997 and intense on 21-22 October 1999.

\subsection{Minima values and their UTs of model SymH* profiles}

As was done for the energy content time series, a distillation of the results in Figs. 8 and 9 can be made by only considering the magnitude and timing of the peak. Figure 12 presents the minimum SymH* values of the modeled SymH* profiles shown in Figs. 8 and 9 during the 6-7 November 1997 storm for the four boundary conditions (BC1-BC4) for the 
October 21-22, 1999 storm, IMPTAM model

\begin{tabular}{|l|l|}
\hline \multicolumn{1}{|c|}{ E field } & Boundary conditions \\
VS: Volland-Stern & BC1: Maxwell at $6.6 \mathrm{Re}, \mathrm{n}=0.5 \mathrm{~cm}^{-3}, \mathrm{~T}=5 \mathrm{keV}$ \\
Boyle: Boyle et al. (1997) & $\mathrm{BC2}$ : kappa at 6.6 Re, $\mathrm{n}, \mathrm{T}_{\|}$and $\mathrm{T}_{\perp}$ from LANL \\
\hline
\end{tabular}
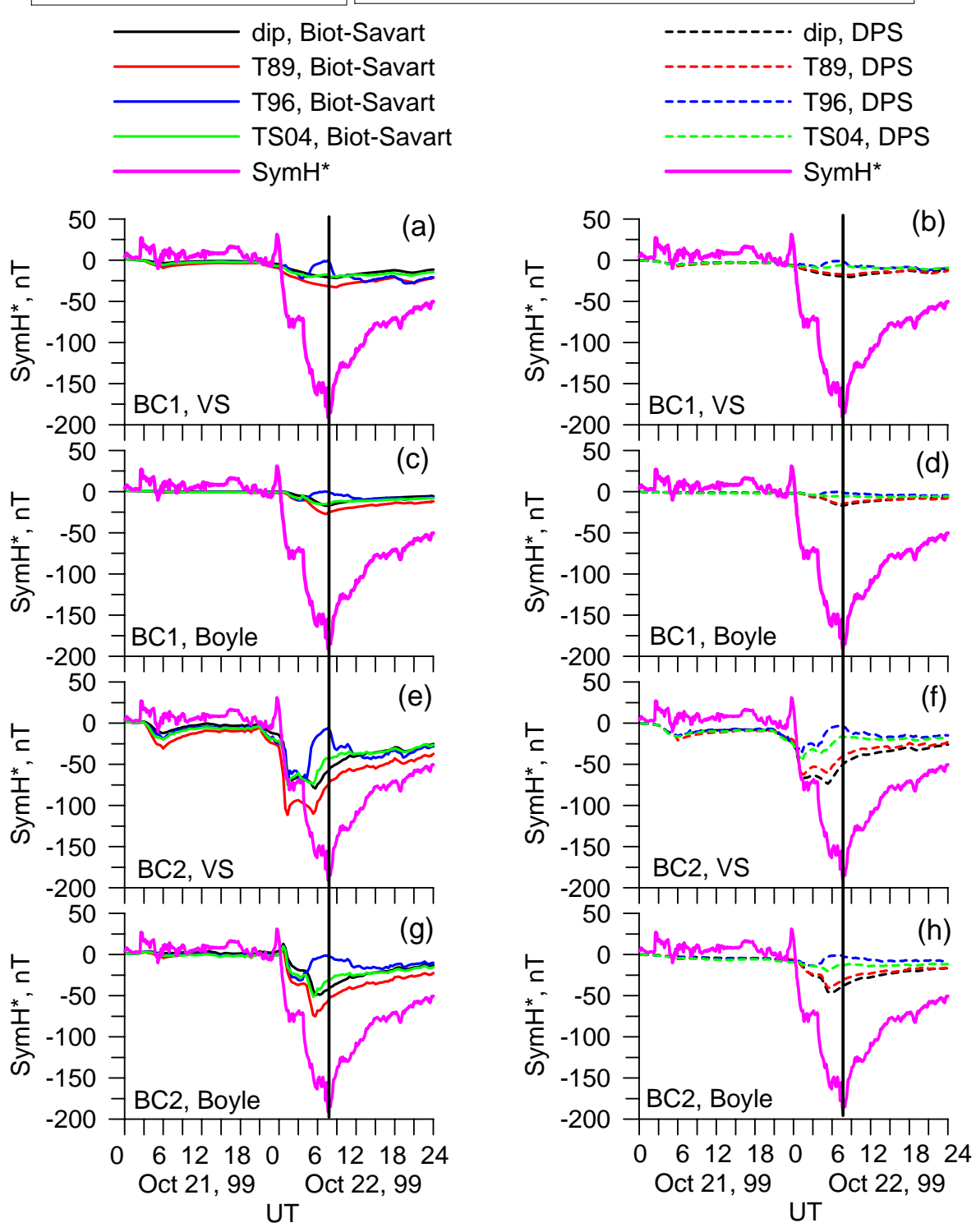

Fig. 10. Similar to Fig. 8 but for 21-22 October 1999 storm.

Volland-Stern (open diamonds) and Boyle (black diamonds) electric fields as dependent on the four magnetic field representations (dipole, T89, T96, and TS04). Figures in the left column were obtained by using the Biot-Savart law and figures in the right column by using the DPS relation for SymH* $^{*}$ calculations. Purple horizontal lines mark the observed SymH* minimum value of $-100 \mathrm{nT}$.

Figure 13 presents, similarly to Fig. 12, the minimum SymH* $^{*}$ values of the modeled SymH* profiles shown in Figs. 10 and 11 during 21-22 October 1999 storm. Purple

horizontal lines mark the observed $\mathrm{SymH}^{*}$ minimum value of $-200 \mathrm{nT}$.

Figure 14 presents the UTs of the minimum SymH* values of the modeled SymH* profiles shown in Figs. 8 and 9 during 6-7 November 1997 storm in terms of the difference $\Delta \mathrm{UT}_{\mathrm{SymH}}=\mathrm{UT}\left(\mathrm{SymH}_{\text {minmod }}\right)-\mathrm{UT}\left(\mathrm{SymH}_{\min }\right)$ between the UT, when the minimum of the modeled SymH* profile is reached during the storm $\mathrm{UT}\left(\mathrm{SymH}_{\text {minmod }}\right)$ and the UT, when the observed $\mathrm{SymH}^{*}$ index dropped to its minimum value $\mathrm{UT}\left(\mathrm{SymH}_{\mathrm{min}}\right)$. Negative values of $\Delta \mathrm{UT}_{\mathrm{SymH}}$ mean 
October 21-22, 1999 storm, IMPTAM model

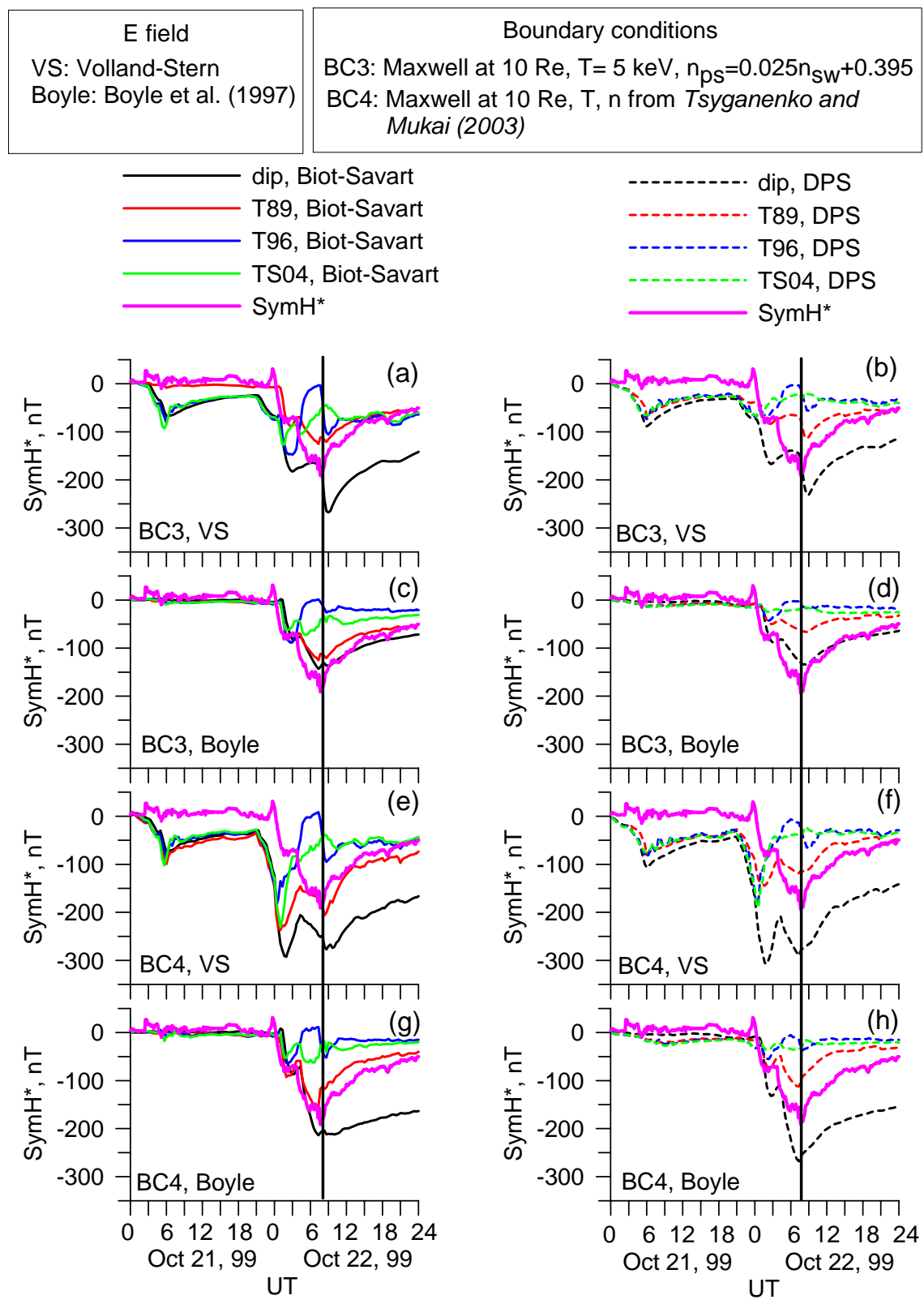

Fig. 11. Similar to Fig. 9 but for 21-22 October 1999 storm.

before and positives values mean after the storm maximum as defined by the UT of SymH* minimum, respectively.

Figure 15 presents the UTs of the minimum SymH* values of the modeled SymH* profiles shown in Figs. 10 and 11 during 21-22 October 1999 storm, similar to Fig. 14.

Table 4 highlights the results of the minimum values and their UTs of modeled SymH* computed using the DesslerParker-Sckopker DPS relation and Biot-Savart integration for both storm events, moderate on 6-7 November 1997 and intense on 21-22 October 1999.

\section{Discussion and conclusions}

The evolution of the storm-time ring current energy profiles, peak magnitudes and their UT differences were modeled by the Inner Magnetosphere Particle Transport and Acceleration model (IMPTAM), developed in Ganushkina et al. (2001, 2005, 2006). Four different magnetic field and two electric field representations and four boundary conditions were used in the simulations. The modeling was performed for two storm events, one moderate (SymH minimum of $-120 \mathrm{nT}$ ) on 6-7 November 1997, and one intense (SymH 
November 6-7, 1997 storm, IMPTAM model

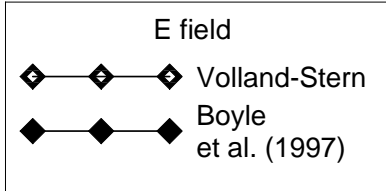

Boundary conditions
BC1: Maxwell at $6.6 \mathrm{Re}, \mathrm{n}=0.5 \mathrm{~cm}^{-3}, \mathrm{~T}=5 \mathrm{keV}$
$\mathrm{BC} 2$ : kappa at $6.6 \mathrm{Re}, \mathrm{n}, \mathrm{T}_{\|}$and $\mathrm{T}_{\perp}$ from $\mathrm{LANL}$
BC3: Maxwell at $10 \mathrm{Re}, \mathrm{T}=5 \mathrm{keV}, \mathrm{n}_{\mathrm{ps}}=0.025 \mathrm{n}_{\mathrm{Sw}}+0.395$
BC4: Maxwell at $10 \mathrm{Re}, \mathrm{T}, \mathrm{n}$ from Tsyganenko and Mukai (2003)

SymH from DPS (e)

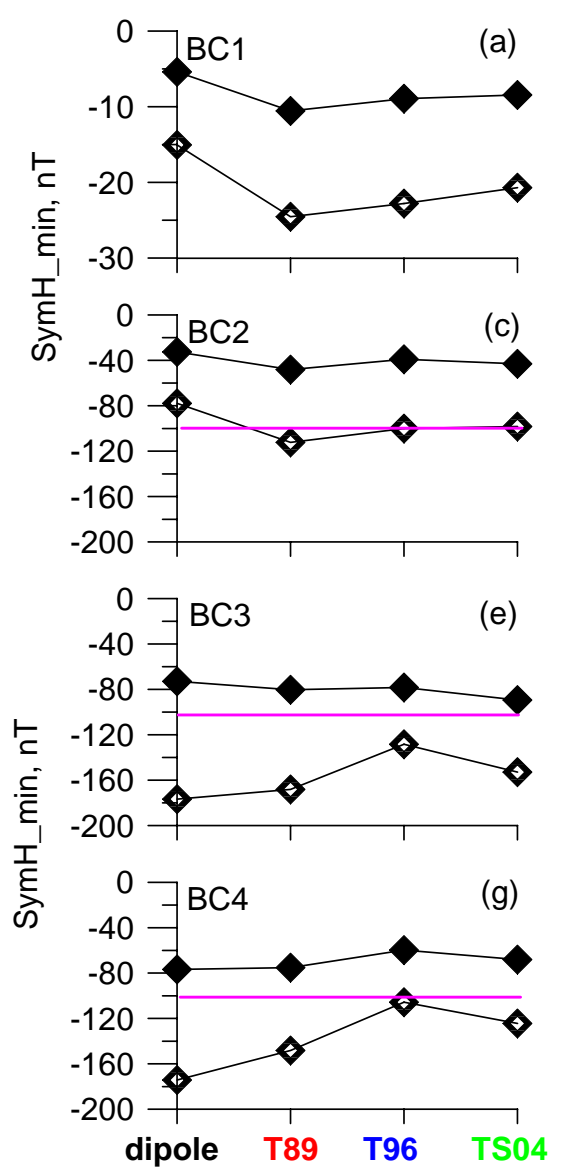

(a)
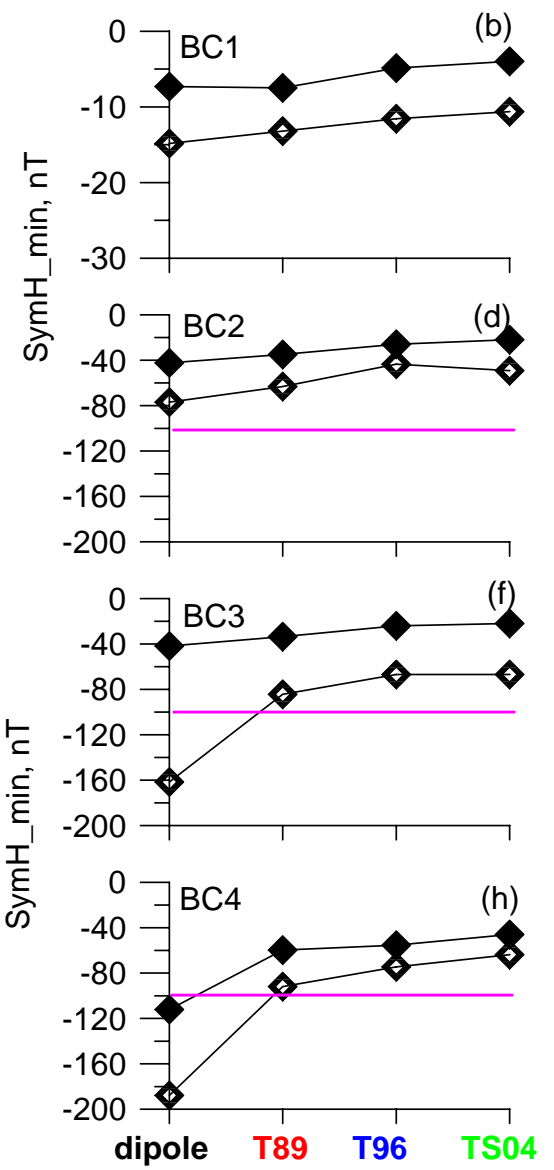

Fig. 12. Minimum SymH* values of the modeled SymH* profiles shown in Figs. 8 and 9 during 6-7 November 1997 storm. Purple horizontal lines mark the observed $\mathrm{SymH}^{*}$ minimum value.

minimum of $-230 \mathrm{nT}$ ) on 21-22 October 1999. In addition, SymH* was computed from the results by two methods, the Dessler-Parker-Sckopke relation (DPS) and Biot-Savart's law. We used varying but prescribed electric and magnetic fields, in which protons move, not taking into account selfconsistency. It was done especially to investigate how much the modeled ring current depends on the background magnetic and electric fields and boundary conditions used in simulations and, correspondingly, how much can physical conclusions made from simulations be dependent on them.

In our modeling the magnetosphere was empty at the beginning. The filling of the inner magnetosphere with particles coming from the plasma sheet is seen for all combinations of magnetic and electric fields and boundary conditions.
There is no dependence on the choice of magnetic field for given electric field and boundary conditions. For the moderate storm (Fig. 2) when using the Boyle field, the ring current energy profiles show peaks corresponding to two small minima around 06:00 and 18:00 UT on 6 November in the observed SymH* index for all magnetic field representations and boundary conditions but with the VS field the profiles are flat. The Boyle field depends on IMF and solar wind velocity $V_{\mathrm{x}}$, and their variations are reflected in the modeled profiles. For the intense storm (Fig. 3), the peak in the ring current energy profile before the storm seen with the VS field does not correspond to any significant dips in $\mathrm{SymH}^{*}$ but is explained by the Kp-dependence of the VS field (observed $\mathrm{Kp}$ increases to 5 around 06:00 UT on 21 October). For the 
October 21-22, 1999 storm, IMPTAM model

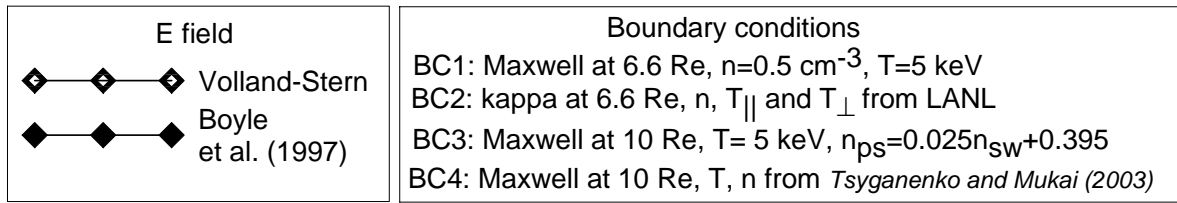

SymH from Biot-Savart
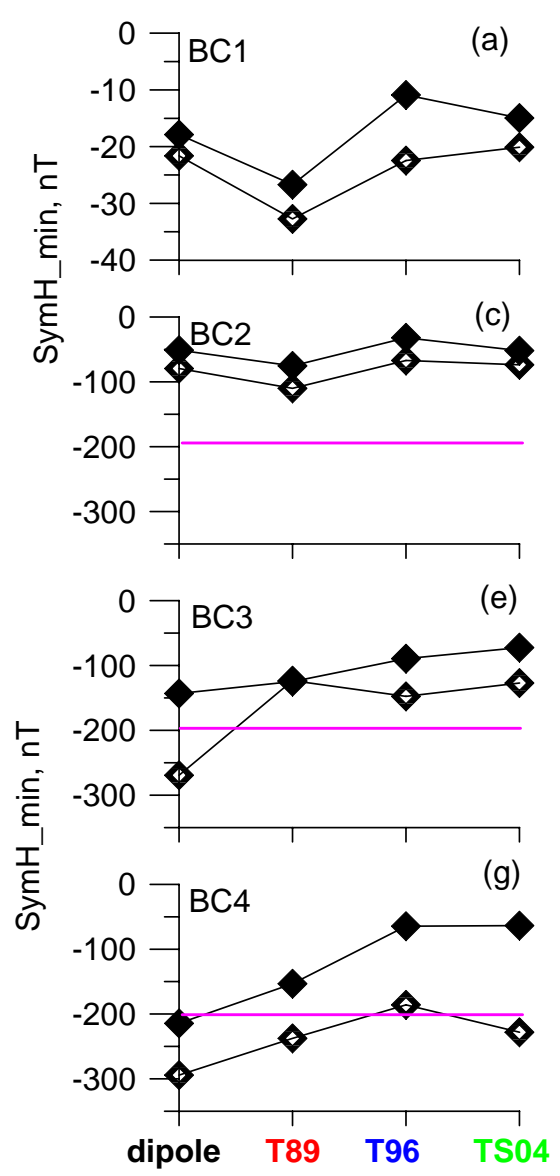

SymH from DPS
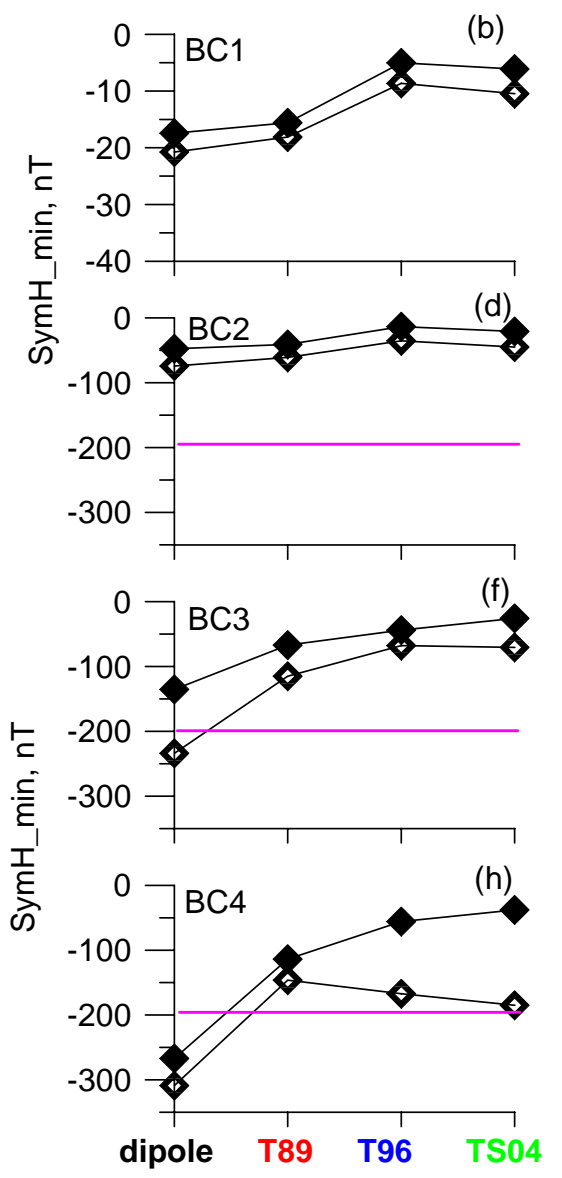

Fig. 13. Minimum SymH* values of the modeled SymH* profiles shown in Figs. 10 and 11 during 21-22 October 1999 storm. Purple horizontal lines mark the observed $\mathrm{SymH}^{*}$ minimum value.

moderate storm, the magnitude of the ring current energy accumulated during the modeling period before the storm does not depend much on the location of the model boundary (at 6.6 or at $10 R_{\mathrm{E}}$ ). It is roughly true for the intense storm too, except for the Kp-dependent peak.

The time when the ring current energy starts to increase sharply at the storm beginning does not depend on magnetic field used and coincides quite well with the observed SymH* $^{*}$ decrease for the Boyle field but occurs $3 \mathrm{~h}$ earlier for the VS field. Further evolution of the ring current energy profiles (in shape, magnitudes, peak(s) locations) strongly depend on the background fields and boundary conditions used. Less realistic magnetic field configurations (dipole and T89) give larger values than more realistic ones (T96 and TS04). Changing from stationary BC1 to LANL timedependent $\mathrm{BC} 2$ boundary condition at $6.6 R_{\mathrm{E}}$ resulted in a factor of 5 increase in the ring current energy. From one side, temporal variations of the source cause more particles to be trapped and added to the ring current energy. From the other side, $\mathrm{BC} 1$ and $\mathrm{BC} 2$ are different in terms of the shape of the distribution (BC1 has Maxwellian and BC2 kappa distribution) and also their average parameters are not the same ( $n=0.5 \mathrm{~cm}^{-3}$ and $T=5 \mathrm{keV}$ for BC1 and $(<T>=7.8 \mathrm{keV}$ and $\langle n\rangle=0.97 \mathrm{~cm}^{-3}$ for $\mathrm{BC} 2$ ). Not only temporal variations but also the magnitudes of number density are important for the ring current energy increase (Kozyra et al., 1998; Chen et al., 2000; Ebihara et al., 2005). With the temperature it is not straightforward, since a cold dense plasma sheet can 
November 6-7, 1997 storm, IMPTAM model

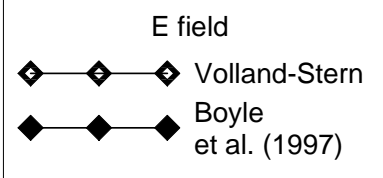

Boundary conditions
BC1: Maxwell at $6.6 \mathrm{Re}, \mathrm{n}=0.5 \mathrm{~cm}^{-3}, \mathrm{~T}=5 \mathrm{keV}$
BC2: kappa at $6.6 \mathrm{Re}, \mathrm{n}, \mathrm{T}_{\|}$and $\mathrm{T}_{\perp}$ from $\mathrm{LANL}$
BC3: Maxwell at $10 \mathrm{Re}, \mathrm{T}=5 \mathrm{keV}, \mathrm{n}_{\mathrm{ps}}=0.025 \mathrm{n}_{\mathrm{Sw}}+0.395$
BC4: Maxwell at $10 \mathrm{Re}, \mathrm{T}, \mathrm{n}$ from Tsyganenko and Mukai (2003) $^{+0}$

SymH from Biot-Savart

SymH from DPS

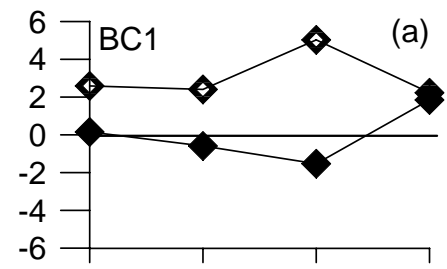

(a)
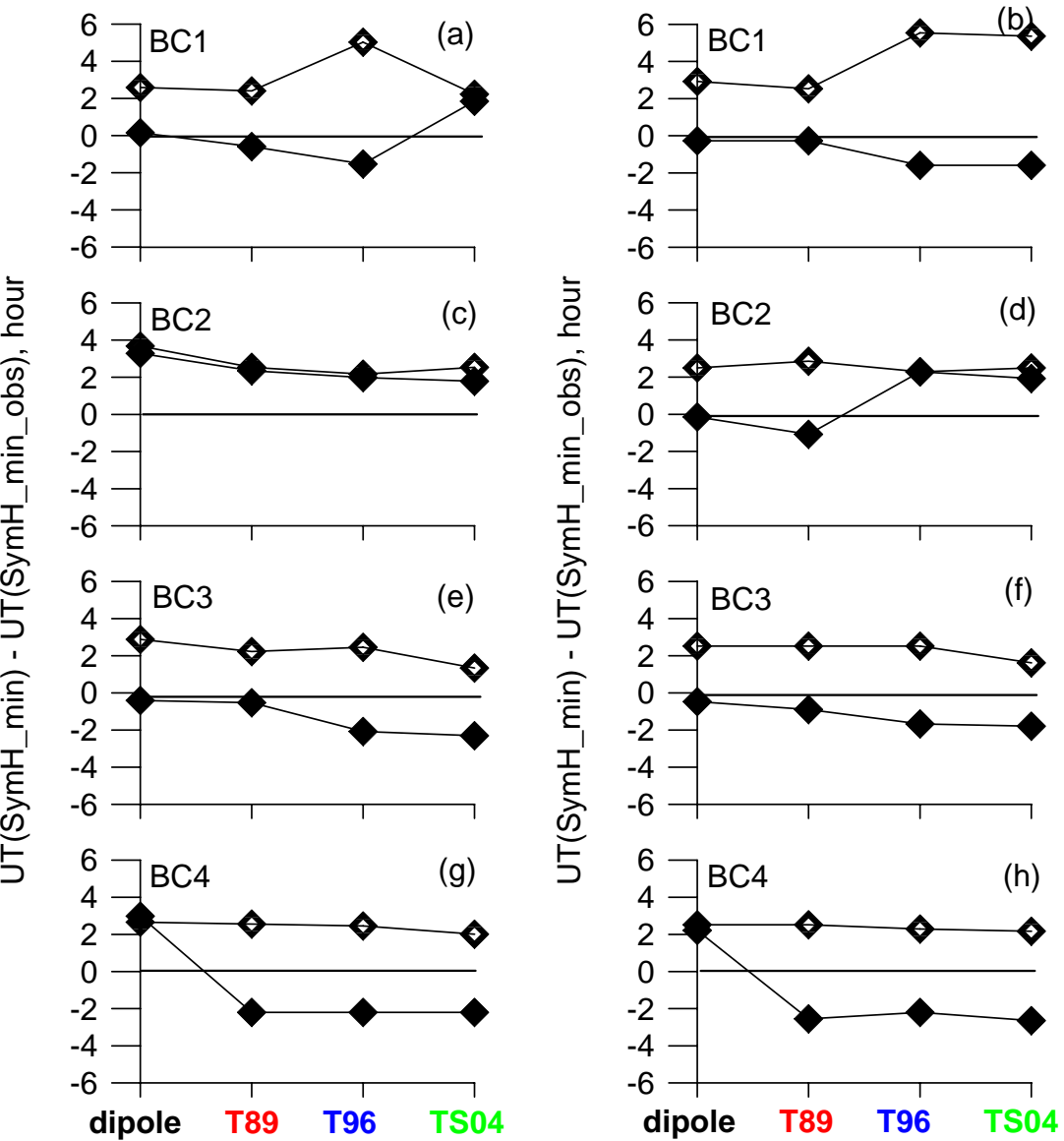

Fig. 14. UTs of the minimum SymH* values of the modeled SymH* profiles shown in Figs. 8 and 9 during 6-7 November 1997 storm in terms of the difference $\Delta \mathrm{UT}_{\mathrm{SymH}}$ (see the text).

lead to an enhanced ring current (Chen et al., 2007; Lavraud and Jordanova, 2007).

With moving the model boundary to $10 R_{\mathrm{E}}$, additional increase by a factor of 1.5 for the moderate storm and a factor of about 2 for the intense storm is detected. This is consistent with previous results by Zheng et al. (2010) that a source that is farther away from the Earth leads to a stronger ring current. Setting the model boundary outside of $6.6 R_{\mathrm{E}}$ gives a possibility to take into account the particles in the transition region (between dipole and stretched field lines) forming the partial ring current and near-Earth tail current in that region. The dipole field produced significantly larger ring current energy compared to other magnetic fields. Assuming the magnetic field to be a dipole inside $10 R_{\mathrm{E}}$ provides easier access of plasma sheet particles to the ring current region.

One of the main indicators that a modeler has succeeded in ring current modeling is the ability of the modeled SymH* index to follow the observed one. In this study, the commonly used DPS relation always gives smaller absolute SymH* values than those calculated using Biot-Savart's law for both moderate and intense storms for all magnetic field models and boundary conditions, except for dipole field. For both storms, when using the DPS relation, the largest absolute $\mathrm{SymH}^{*}$ values are obtained when using a dipole field and the smallest, when using T96 field, with T89 and TS04 in between (Figs. 8, 9 and Figures 10,11), which corresponds 
October 21-22, 1999 storm, IMPTAM model

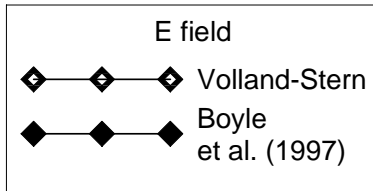

Boundary conditions
BC1: Maxwell at $6.6 \mathrm{Re}, \mathrm{n}=0.5 \mathrm{~cm}^{-3}, \mathrm{~T}=5 \mathrm{keV}$
BC2: kappa at $6.6 \mathrm{Re}, \mathrm{n}, \mathrm{T}_{\|}$and $\mathrm{T}_{\perp}$ from LANL
BC3: Maxwell at $10 \mathrm{Re}, \mathrm{T}=5 \mathrm{keV}, \mathrm{n}_{\mathrm{ps}}=0.025 \mathrm{n}_{\mathrm{Sw}}+0.395$
BC4: Maxwell at $10 \mathrm{Re}, \mathrm{T}, \mathrm{n}$ from Tsyganenko and Mukai (2003)

SymH from Biot-Savart

SymH from DPS
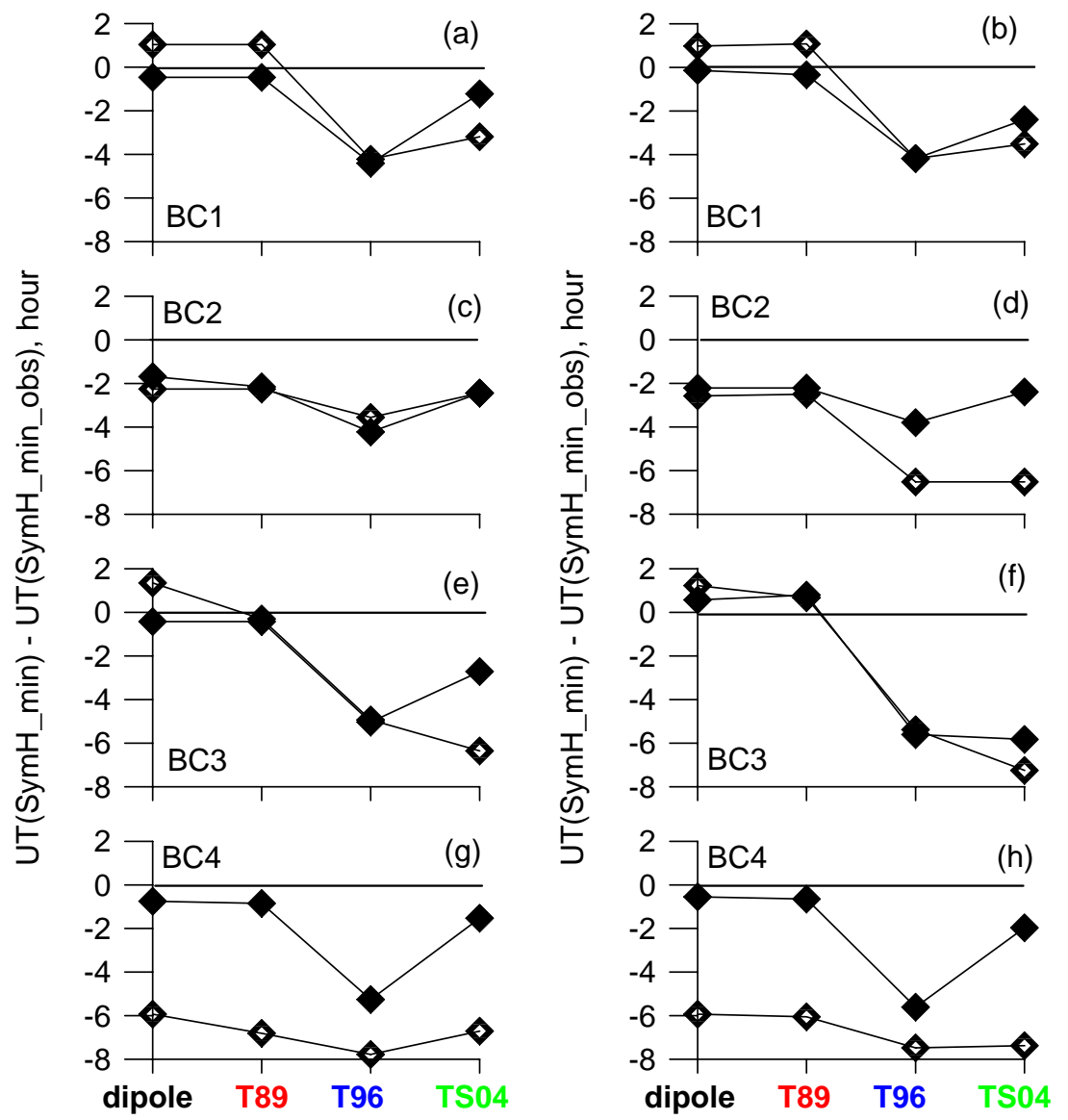

Fig. 15. UTs of the minimum SymH* values of the modeled SymH* profiles during 21-22 October 1999 storm, similar to Fig. 14.

to the ring current energy profiles (Figs. 2 and 3). When the Biot-Savart method is used, the order is different: the largest absolute SymH* $^{*}$ values are with the T89 field, and absolute $\mathrm{SymH}^{*}$ magnitudes calculated with a dipole can be close to those of T96 and TS04 with some variations. This reflects the fact that when using Biot-Savart's law, the modeled SymH* is not assumed to come from the ring current energy only.

Figure 16 shows the differences $\Delta$ SymH $^{*}$ between SymH* calculated by Biot-Savart's law and DPS relation for both storms, for all model combinations and for LANL timedependent $\mathrm{BC} 2$ at $6.6 R_{\mathrm{E}}$ and $\mathrm{BC} 4$ at $10 R_{\mathrm{E}}$. It can be seen that for dipole magnetic field the difference are mainly positive before the storm and for the beginning storm main phase for both VS and Boyle electric fields and BC2 and BC4. It means that DPS gives smaller SymH* values (larger in absolute values) than Biot-Savart's law. Here we need to remember how DPS is calculated and with what assumptions following Liemohn (2003) study.

DPS relation was derived for the dipole field $\left(\frac{\Delta B}{B_{E}}=\right.$ $-\frac{2 E_{\mathrm{RC}}}{3 U_{E}}$, where $B_{E}$ is the equatorial surface magnetic field strength of the Earth's dipole and $U_{E}$ is the magnetic energy of the dipole field beyond the Earth's surface. Liemohn (2003) did their calculations in the dipole magnetic field and "found that the truncation current is implicitly included in the DPS relation whenever the plasma pressure is nonzero at the outer boundary. This truncation current is introduced because the DPS relation assumes that all of the plasma pressure is contained within the integration volume. The 

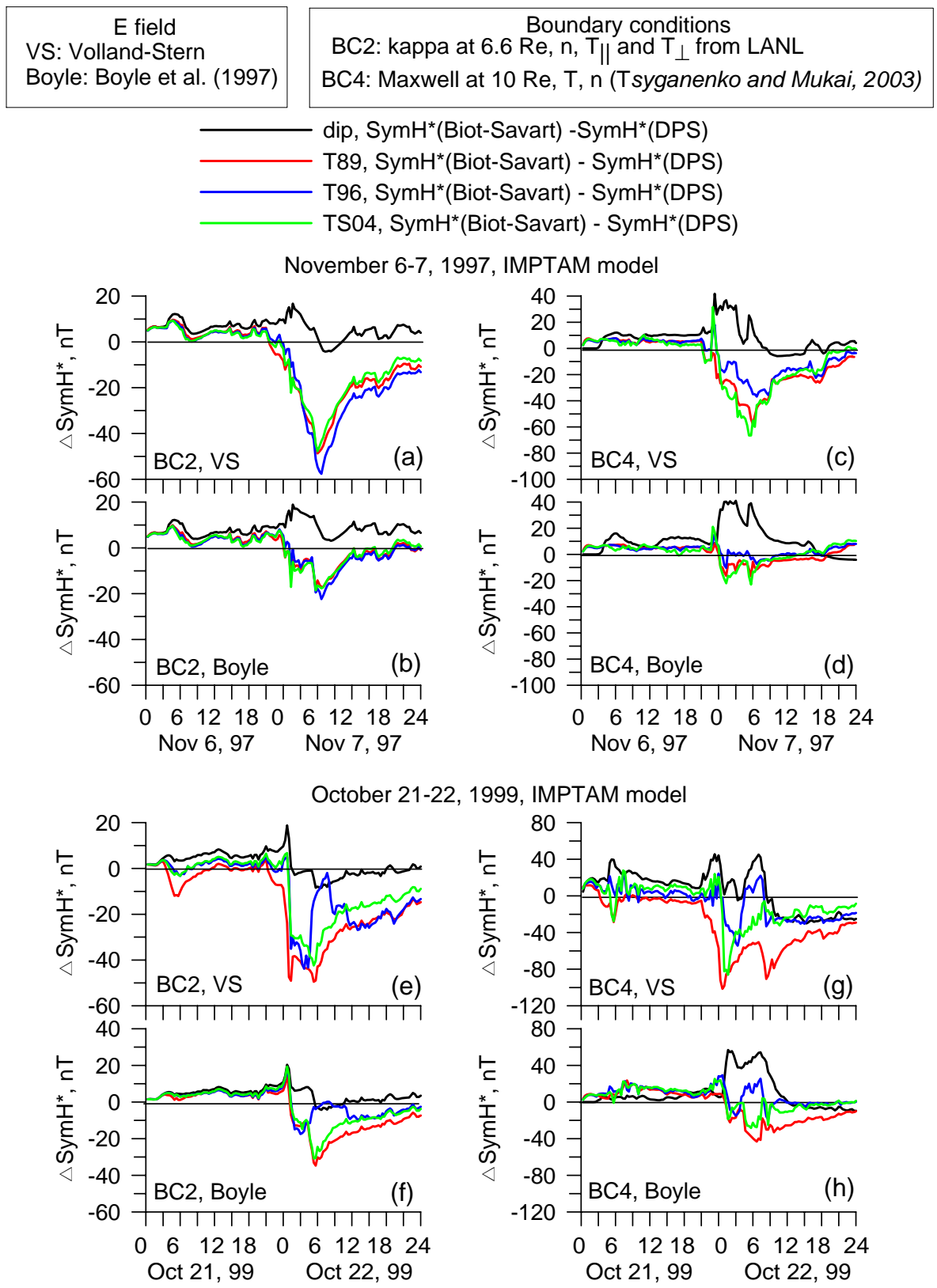

Fig. 16. Differences between model SymH* calculated by Biot-Savart's law and DPS relation for moderate 6-7 November 1997 and intense 21-22 October 1999 storm events.

truncation current implicitly included in the DPS relation is, in some qualitative sense, accounting for the tail current or any current beyond the outer boundary." When we calculate SymH* by Biot-Savart's law for dipole magnetic field, we do not include this current, so $\mathrm{SymH}^{*}$ calculated by BiotSavart's law is larger (smaller in absolute values) than that by DPS.

When we calculate SymH* by Biot-Savart's law in Tsyganenko magnetic fields, we include the effect from the nearEarth tail current by including the effect from the stretched magnetic field lines for both boundaries at 6.6 and $10 R_{\mathrm{E}}$.
DPS relation also contains some sort of contribution from other currents than the ring current but incorrectly. Especially, when the ring current energy used in the DPS relation is calculated following particles in the nondipolar magnetic field, but other magnitudes are still obtained from the dipole field.

As can be noticed in Fig. 16, differences, when $\mathrm{SymH}^{*}$ is calculated by both methods in nondipolar magnetic fields are negative (Biot-Savart's law gives smaller values of SymH* than DPS relation) and somewhat close in magnitudes for all three Tsyganenko fields for given electric field and boundary 
Table 3. Model-dependent storm-time ring current: modeled SymH* index.

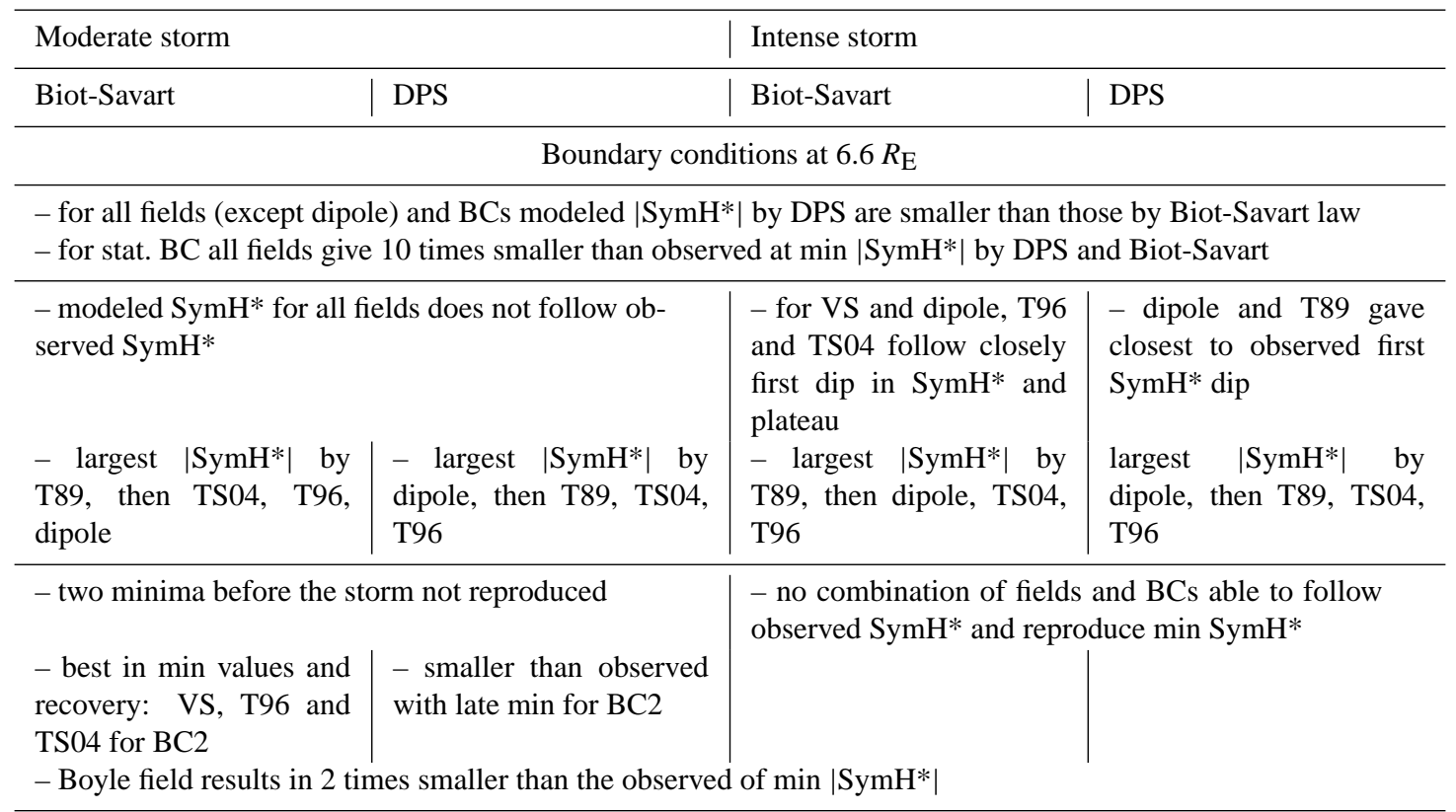

Boundary conditions at $10 R_{\mathrm{E}}$

\begin{tabular}{|c|c|c|}
\hline $\begin{array}{l}\text { - for VS and dipole and } \\
\text { T89 }\left|\mathrm{SymH}^{*}\right| \quad 2 \text { times } \\
\text { larger }\end{array}$ & $\begin{array}{l}\text { - for VS and all magnetic } \\
\text { fields } 2 \text { times smaller of } \\
\mid \text { SymH*| } \\
-\quad \text { exception: dipole: } \\
60 \text { nT min } \\
\text { overestimate }\end{array}$ & $\begin{array}{l}\text { - except for BC3 and Boyle, dipole largerly overes- } \\
\text { timates }\left|\mathrm{SymH}^{*}\right|\end{array}$ \\
\hline $\begin{array}{l}\text { - for all magnetic fields an } \\
\text { - for Boyle and all } \\
\text { magnetic fields SymH* } \\
\text { follow observed before } \\
\text { storm and SymH drop, } \\
\text { not storm max }\end{array}$ & $\begin{array}{l}\text { VS, SymH* drops } 2 \mathrm{~h} \text { earli } \\
- \text { only }-40 \mathrm{nT} \text { by dipole, } \\
\text { compared to }-100 \mathrm{nT}\end{array}$ & 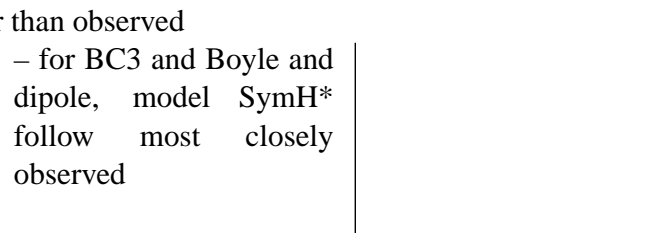 \\
\hline
\end{tabular}

condition for moderate 6-7 November 1997 storm (Fig. 16ad). For intense storm on 21-22 October 1999 (Fig. 16e-h), the situation is generally the same, except for the variations when using T96 model discussed previously in the present paper.

Different combinations of fields and boundary conditions result in different modeled SymH*. For the moderate storm and using Biot-Savart's law, the closest modeled SymH* profiles to the observed one were obtained for LANL timedependent boundary conditions at $6.6 R_{\mathrm{E}}$ for VS electric field and T96 and TS04 magnetic fields (Fig. 8e). In Fig. 8, none of the modeled $\mathrm{SymH}^{*}$ profiles follow the sharp decrease (they are not sharp enough) in the observed SymH* and they eventually reach minimum $2-3 \mathrm{~h}$ after the observed minimum. With Biot-Savart's law inside $10 R_{\mathrm{E}}$, the modeled absolute SymH* values are overestimated for the VS field and all magnetic fields. The VS field is not suitable here, and also the boundary conditions at $10 R_{\mathrm{E}}$ are far from being realistic, even though the plasma sheet moments depend on IMF and solar wind parameters.

At the same time, the modeled SymH* profiles follow much better the observed sharp decrease, even if they start to drop earlier and $\mathrm{SymH}^{*}$ minima do not coincide (Fig. 9a, e). When setting the model boundary at $10 R_{\mathrm{E}}$ and using Tsyganenko magnetic fields, we include the effect from the nearEarth tail current from the stretched magnetic field lines. The results are in agreement with the modeling efforts by Ganushkina et al. (2004). They have shown that during moderate storms the tail current intensifies first and tracks the drop in the Dst index. The ring current develops more slowly, and then stays at an increased level longer than the tail current. The Boyle field works better, and agreement for SymH* is best for the T89 magnetic field and BC3. For the DPS relation, only the dipole field gave unrealistically large absolute 
Table 4. Model-dependent storm-time ring current: minimum values and their UTs of model SymH*.

\begin{tabular}{|c|c|c|c|}
\hline \multicolumn{2}{|l|}{ Moderate storm } & \multicolumn{2}{|l|}{ Intense storm } \\
\hline Biot-Savart & DPS & Biot-Savart & DPS \\
\hline \multicolumn{4}{|c|}{ Minimum values of model SymH* } \\
\hline $\begin{array}{l}\text { - VS produced larger value } \\
\text { - for stationary } \mathrm{BC} \text { min } \\
-5 \mathrm{nT} \\
- \text { closest to observed } \\
\text { min }\left|\mathrm{Sym} \mathrm{H}^{*}\right| \text { for } \mathrm{BC} 2 \text { at } \\
6.6 R_{\mathrm{E}} \text { and } \mathrm{VS} \text { and } \mathrm{T} 96 \\
\text { and TS04 } \\
\text { - not much dependence or } \\
|\mathrm{SymH}| \text { with Boyle field } \\
-\quad \text { significant min } \\
\mid \text { SymH } \\
\text { for VS and all magnetic } \\
\text { fields for BC at } 10 R_{\mathrm{E}}\end{array}$ & $\begin{array}{l}\text { of min }\left|\mathrm{Sym} \mathrm{H}^{*}\right| \text { than Boyl } \\
\text { ymH* are in }-25 \text { to } \\
\text { - no close to observed } \\
\text { min SymH*, best for } \\
\mathrm{VS} \text { and } \mathrm{T} 89 \text { with } \mathrm{BC} \text { at } \\
10 R_{\mathrm{E}} \\
\text { magnetic field for min } \\
\text { - significant min } \\
|\mathrm{SymH}| \text { overestimates } \\
\text { for } \mathrm{VS} \text { and dipole for BC } \\
\text { at } 10 R_{\mathrm{E}}\end{array}$ & $\begin{array}{l}\text { for all magnetic fields and } \mathrm{E} \\
\text { - for } \mathrm{BC} \text { at } 6.6 R_{\mathrm{E}} \min \mid \mathrm{S} \\
\text { served, } 10 \text { times } \mathrm{BC} 1 \text { and } \\
\text { - close to observed for } \\
\text { VS, T96 and TS04, and } \\
\text { dipole and Boyle for BC4 }\end{array}$ & $\begin{array}{l}\text { Cs } \\
\text { ImH*| smaller than ob- } \\
\text { times for BC2 } \\
\text { - closest to observed for } \\
\text { VS and T96 and TS04 for } \\
\text { BC4 }\end{array}$ \\
\hline \multicolumn{4}{|c|}{ UT differences of model minimum SymH* } \\
\hline $\begin{array}{l}\text { - for VS min } \mathrm{SymH}^{*} 2-5 \\
\text { magnetic fields and BCs } \\
\text { - smallest } \Delta \mathrm{UT}_{\mathrm{SymH}} \text { for } \\
\text { Boyle and dipole and } \mathrm{T} 89 \\
\text { for } \mathrm{BC} 1 \text { and } \mathrm{BC} 3\end{array}$ & $\begin{array}{l}\text { after observed for all } \\
\text { - smallest } \Delta \mathrm{UT}_{\text {SymH }} \text { for } \\
\text { Boyle and dipole and } \mathrm{T} 89 \\
\text { for } \mathrm{BC} 1, \mathrm{BC} 2 \text { and } \mathrm{BC} 3\end{array}$ & \multicolumn{2}{|c|}{$\begin{array}{l}\text { - for stationary BC similar for VS and Boyle and all } \\
\text { magnetic fields } \\
\text { - close to zero } \Delta \mathrm{UT}_{\mathrm{SymH}} \text { for Boyle and dipole and } \\
\mathrm{T} 89 \text { for BC1, BC3 and BC4 }\end{array}$} \\
\hline \multicolumn{2}{|c|}{$\begin{array}{l}\text { - at } 10 R_{\mathrm{E}} \text { for T96 and TS04 modeled storm max } \\
\text { occurred } 2 \mathrm{~h} \text { for Boyle field }\end{array}$} & \multicolumn{2}{|c|}{$\begin{array}{l}\text { - at } 10 R_{\mathrm{E}} \text { for T96 and TS04 modeled storm max } \\
\text { occurred much earlier ( } 6 \text { to } 8 \mathrm{~h} \text { ) for VS and Boyle }\end{array}$} \\
\hline
\end{tabular}

$\mathrm{SymH}^{*}$ values due to the easier access of plasma sheet particles to the ring current region.

For the intense storm, no combination was able to reproduce the two-step decrease in the observed SymH*, only one combination (of Boyle, T89 and BC4 boundary conditions at $10 R_{\mathrm{E}}$ ) resulted in relatively close magnitudes. Note that using the T96 and TS04 magnetic fields resulted in very unrealistic SymH* profiles with peaks even at the observed SymH* minimum. The magnetic field configuration in the inner magnetosphere during the intense storm on 21-22 October 1999 was modeled by using different magnetic fields by Kalegaev et al. (2005). It was shown that the Tsyganenko T02 field (Tsyganenko, 2002) underestimated the $B_{\mathrm{Z}}$ component significantly (by $100 \mathrm{nT}$ ) at the storm maximum compared to GOES 8 and GOES 10 measurements. GOES 8 was around midnight and GOES 10 was moving toward midnight in the dusk sector. This indicates that precautions must be taken when using realistic yet empirical magnetic fields in real storm event modeling. It is always good to compare the observed magnetic field with the modeled one for an event to be modeled, when globally applying a magnetic field configuration to the magnetosphere.
In general, the structure of the observed SymH* profiles during the intense storm is better reproduced than during the moderate storm, even using the DPS method, although the actual magnitudes are not the same. It follows the previous modeling results by Ganushkina et al. (2004) that during intense storms, the tail current is intensified, but the main contribution comes from the ring current. Note that Ganushkina et al. (2004) modeling was conducted using the eventoriented magnetospheric magnetic field representation technique based on actual magnetic field measurements for several events, and in the present paper we employ the particle model IMPTAM.

Keeping the points discussed above in mind the conclusions are as follows:

1. Different combinations of the magnetic and electric fields and boundary conditions result in very different modeled ring current, and, therefore, the physical conclusions based on simulation results can differ significantly.

2. A time-dependent model boundary outside of $6.6 R_{\mathrm{E}}$ gives a possibility to take into account the particles in the transition region (between dipole and stretched field 
lines) forming a partial ring current and near-Earth tail current in that region.

3. Calculating the model SymH* by Biot-Savart's law instead of the widely used Dessler-Parker-Sckopke (DPS) relation in nondipolar magnetic field gives larger and more realistic values, since the effect from the nearEarth tail current is included by the presence of the stretched magnetic field lines.

4. Particle modeling results are in agreement with previous magnetic field modeling results by Ganushkina et al. (2004) that the current systems behave differently for moderate and intense storms. The tail current is very important for moderate storms.

For ring current modeling to be correct, the model boundary needs to be placed outside of $6.6 R_{\mathrm{E}}$, and the model $\mathrm{SymH}^{*}$ index has to be computed directly from the modeled current, using Biot-Savart's law.

Acknowledgements. The authors gratefully acknowledge the support of this work by NASA through grants NNG05GM48G, NNX07AL88G, and NNX08AQ15G, by NSF through grant ATM0802705, and by the LANL-IGPP minigrant program. We thank $\mathrm{K}$. Ogilvie and R. Lepping for the use of WIND data in this paper, World Data Center C2 for Geomagnetism, Kyoto, for the provisional $\mathrm{AE}, \mathrm{Kp}, \mathrm{SymH}$, and Dst indices data. The data were obtained from the Coordinated Data Analysis Web (CDAWeb). Natalia Ganushkina's work was partly supported by the Academy of Finland. The part of the research leading to these results has received funding from the European Union Seventh Framework Programme (FP7/2007-2013) under grant agreement no. 262468. We thank H. Koskinen for very useful discussions.

Topical Editor I. A. Daglis thanks two anonymous referees for their help in evaluating this paper.

\section{References}

Alexeev, I. I., Kalegaev, V. V., Belenkaya, E. S., Bobrovnikov, S. Y., Feldstein, Y. I., and Gromova, L. I.: Dynamic model of the magnetosphere: Case study for January 9-12, 1997, J. Geophys. Res., 106, 5683-25693, 2001.

Angelopoulos, V., Temerin, M., Roth, I., Mozer, F. S., Weimer, D., and Hairston, M. R.: Testing global storm-time electric field models using particle spectra on multiple spacecraft, J. Geophys. Res., 107, 1194, doi:10.1029/2001JA900174, 2002.

Arykov, A. A. and Maltsev, Yu. P.: Contribution of various sources to the geomagnetic storm field, Geomag. Aeron., 33, 67-74, 1993.

Bame, S. J., McComas, D. J., Thomsen, M. F., Barraclough, B. L., Elphic, R. C., Glore, J. P., Gosling, J. T., Chavez, J. C., Evans, E. P., and Wymer, F. J.: Magnetospheric plasma analyzer for spacecraft with constrained resources, Rev. Sci. Instr., 64, 1026-1033, 1993.

Boyle, C., Reiff, P., and Hairston, M.: Empirical polar cap potentials, J. Geophys. Res., 102, 111-125, 1997.
Burton, R. K., McPherron, R. L., and Russell, C. T.: An empirical relationship between interplanetary conditions and Dst, J. Geophys. Res., 80, 4204-4214, 1975.

Campbell, W. H.: The field levels near midnight at low and equatorial geomagnetic stations, J. Atmos. Terr. Phys., 35, 1127-1146, 1973.

Carovillano, R. L. and Siscoe, G. L.: Energy and momentum theorems in magnetospheric processes, Rev. Geophys. Space Phys. 11, 289-353, 1973.

Carpenter, D. and Anderson, R.: An ISEE/Whistler Model of Equatorial Electron Density in the Magnetosphere, J. Geophys. Res., 97, 1097-1108, 1992.

Chen, M. W., Schulz, M., Lyons, L. R., and Gorney, D. J.: Stormtime Transport of Ring Current and Radiation Belt Ions, J. Geophys. Res., 98, 3835-3849, 1993.

Chen, M. W., Lyons, L. R., and Schulz, M.: Stormtime ring-current formation: A comparison between single- and double-dip model storms with similar transport characteristics, J. Geophys. Res., 105, 27755-27765, 2000.

Chen, M. W., Liu, S., Schulz, M., Roeder, J. L., and Lyons, L. R.: Magnetically self-consistent ring current simulations during the 19 October 1998 storm, J. Geophys. Res., 111, A11S15, doi:10.1029/2006JA011620, 2006.

Chen, M. W., Wang, C.-P., Schulz, M., and Lyons, L. R.: Solarwind influence on MLT dependence of plasma sheet conditions and their effects on storm time ring current formation, Geophys. Res. Lett., 34, L14112, doi:10.1029/2007GL030189, 2007.

Dessler, A. J. and Parker, E. N.: Hydromagnetic theory of geomagnetic storms, J. Geophys. Res., 64, 2239-2252, 1959.

Dremukhina, L. A., Feldstein, Y. I., Alexeev, I. I., Kalegaev, V. V., and Greenspan, M.: Structure of the magnetospheric magnetic field during magnetic storms, J. Geophys. Res., 104, 2835128360, 1999.

Ebihara, Y. and Ejiri, M.: Simulation study on fundamental properties of the storm-time ring current, J. Geophys. Res., 105, 1584315859, doi:10.1029/1999JA900493, 2000.

Ebihara, Y., Fok, M.-C., Wolf, R. A., Thomsen, M. F., and Moore, T. E.: Nonlinear impact of plasma sheet density on the storm-time ring current, J. Geophys. Res., 110, A02208, doi:10.1029/2004JA010435, 2005.

Ebihara, Y., Fok, M.-C., Blake, J. B., and Fennell, J. F.: Magnetic coupling of the ring current and the radiation belt, J. Geophys. Res., 113, A07221, doi:10.1029/2008JA013267, 2008.

Fok, M.-C., Kozyra, J. U., Nagy, A. F., Rasmussen, C. E., and Khazanov, G. V.: Decay of Equatorial Ring Current Ions and Associated Aeronomical Consequences, J. Geophys. Res., 98, 1938119393, doi:10.1029/93JA01848, 1993.

Fok, M.-C., Wolf, R. A., Spiro, R. W., and Moore, T. E.: Comprehensive computational model of Earth's ring current, J. Geophys. Res., 106, 8417-8424, doi:10.1029/2000JA000235, 2001.

Fok, M.-C., Moore, T. E., Wilson, G. R., Perez, J. D., Zhang, X. X., Brandt, P. C.:Son, Mitchell, D. G., Roelof, E. C., Jahn, J.-M., Pollock, C. J., and Wolf, R. A.: Global ENA Image Simulations, Space Sci. Rev., 109, 77-103, doi:10.1023/B:SPAC.0000007514.56380.fd, 2003.

Fok, M.-C., Horne, R. B., Meredith, N. P., and Glauert, S. A.: Radiation Belt Environment model: Application to space weather nowcasting, J. Geophys. Res., 113, A03S08, doi:10.1029/2007JA012558, 2008. 
Gamayunov, K. V., Khazanov, G. V., Liemohn, M. W., Fok, M.-C., and Ridley, A. J.: Self-consistent model of magnetospheric electric field, ring current, plasmasphere, and electromagnetic ion cyclotron waves: Initial results, J. Geophys. Res., 114, A03221, doi:10.1029/2008JA013597, 2009.

Ganushkina, N. Y., Pulkkinen, T. I., Bashkirov, V. F., Baker, D. N., and Li, X.: Formation of intense nose structures, Geophys. Res. Lett., 28, 491-494, 2001.

Ganushkina, N. Yu., Pulkkinen, T. I., Kubyshkina, M. V., Singer, H. J., and Russell, C. T.: Long-term evolution of magnetospheric current systems during storms, Ann. Geophys., 22, 1317-1334, doi:10.5194/angeo-22-1317-2004, 2004.

Ganushkina, N. Yu., Pulkkinen, T. I., and Fritz, T.: Role of substorm-associated impulsive electric fields in the ring current development during storms, Ann. Geophys., 23, 579-591, doi:10.5194/angeo-23-579-2005, 2005.

Ganushkina, N., Pulkkinen, T. I., Liemohn, M., and Milillo, A.: Evolution of the proton ring current energy distribution during April 21-25, 2001 storm, J. Geophys. Res., 111, A11S08, doi:10.1029/2006JA011609, 2006.

Ganushkina, N. Yu., Liemohn, M. W., Kubyshkina, M. V., Ilie, R., and Singer, H. J.: Distortions of the magnetic field by stormtime current systems in Earth's magnetosphere, Ann. Geophys., 28, 123-140, doi:10.5194/angeo-28-123-2010, 2010.

Greenspan, M. E. and Hamilton, D. C.: A test of the Dessler-ParkerSckopke relation during magnetic storms, J. Geophys. Res., 105, 5419-5430, doi:10.1029/1999JA000284, 2000.

Häkkinen, L. V. T., Pulkkinen, T. I., Nevanlinna, H., Pirjola, R. J., and Tanskanen, E. I.: Effects of induced currents on Dst and on magnetic variations at midlatitude stations, J. Geophys. Res., 107, 1014, doi:10.1029/2001JA900130, 2002.

Hamilton, D. C., Gloeckler, G., Ipavich, F. M., Stridemann, W., Wilken, B., and Kremser, G.: Ring current development during the great geomagnetic storm of February 1986, J. Geophys.Res., 93, 14343-14355, 1988.

Hedin, A.: Extension of the MSIS Thermosphere Model into the Middle and Lower Atmosphere, J. Geophys. Res., 96, 11591172, 1991.

Jaggi, R. K. and Wolf, R. A.: Self-Consistent Calculation of the Motion of a Sheet of Ions in the Magnetosphere, J. Geophys. Res., 78, 2852-2866, doi:10.1029/JA078i016p02852, 1973.

Janev, R. K. and Smith, J. J.: Cross sections for collision processes of hydrogen atoms with electrons, protons, and multiply-charged ions, Atomic and Plasma-Material Interaction Data for Fusion, Int. At. Energ. Agency, 4, 78-79, 1993.

Jordanova, V. K., Kistler, L. M., Kozyra, J. U., Khazanov, G. V., and Nagy, A. F.: Collisional losses of ring current ions, J. Geophys. Res., 101, 111-126, doi:10.1029/95JA02000, 1996.

Jordanova, V. K., Kistler, L. M., Farrugia, C. J., and Torbert, R. B.: Effects of Inner Magnetospheric Convection on Ring Current Dynamics: March 10-12, 1998, J. Geophys. Res., 106, 2970529720, 2001.

Jordanova, V. K., Miyoshi, Y. S., Zaharia, S., Thomsen, M. F., Reeves, G. D., Evans, D. S., Mouikis, C. G., and Fennell, J. F.: Kinetic simulations of ring current evolution during the Geospace Environment Modeling challenge events, J. Geophys. Res., 111, A11S10, doi:10.1029/2006JA011644, 2006.

Jordanova, V. K., Matsui, H., Puhl-Quinn, P. A., Thomsen, M. F., Mursula, K., and Holappa, L.: Ring current development during high speed streams, J. Atmos. Solar-Terr. Phys., 71, 1093-1102, 2009.

Kalegaev, V. V., Ganushkina, N. Y., Pulkkinen, T. I., Kubyshkina, M. V., Singer, H. J., and Russell, C. T.: Relation between the ring current and the tail current during magnetic storms, Ann. Geophys., 23, 523-533, doi:10.5194/angeo-23-523-2005, 2005.

Khazanov, G. V., Liemohn, M. W., Fok, M.-C., Newman, T. S., and Ridley, A. J.: Stormtime particle energization with AMIE potentials, J. Geophys. Res., 109, A05209, doi:10.1029/2003JA010186, 2004.

Kozyra, J. U., Jordanova, V. K., Borovsky, J. E., Thomsen, M. F., Knipp, D. J., Evans, D. S., McComas, D. J., and Cayton, T. E.: Effects of a High-density Plasma Sheet on Ring Current Development during the November 2-6, 1993, J. Geophys. Res., 103, 26285-26305, 1998.

Lavraud, B. and Jordanova, V. K.: Modeling the effects of colddense and hot-tenuous plasma sheet on proton ring current energy and peak location, Geophys. Res. Lett., 34, L02102, doi:10.1029/2006GL027566, 2007.

Lavraud, B., Thomsen, M. F., Borovsky, J. E., Denton, M. H., and Pulkkinen, T. I.: Magnetosphere preconditioning under northward IMF: Evidence from the study of coronal mass ejection and corotating interaction region geoeffectiveness, J. Geophys. Res., 111, A09208, doi:10.1029/2005JA011566, 2006.

Lemon, C., Toffoletto, F., Hesse, M., and Birn, J.: Computing magnetospheric force equilibria, J. Geophys. Res., 108, 1237, doi:10.1029/2002JA009702, 2003.

Li, X., Baker, D. N., Temerin, M., Reeves, G. D., and Belian, R. D.: Simulation of dispersionless injections and drift echoes of energetic electrons associated with substorms, Geophys. Res. Lett., 25, 3763-3766, 1998.

Liemohn, M. W.: Yet another caveat to using the DesslerParker-Sckopke relation, J. Geophys. Res., 108, 1251, doi:10.1029/2003JA009839, 2003.

Liemohn, M. W., Kozyra, J. U., Thomsen, M. F., Roeder, J. L., Lu, G., Borovsky, J. E., and Cayton, T. E.: Dominant role of the asymmetric ring current in producing the stormtime Dst*, J. Geophys. Res., 106, 10883-10904, 2001.

Liemohn, M. W., Kozyra, J. U., Hairston, M. R., Weimer, D. R., Lu, G., Ridley, A. J., Zurbuchen, T. H., and Skoug, R. M.: Consequences of a Saturated Convection Electric Field on the Ring Current, Geophys. Res. Lett., 29, 1348, doi:10.1029/2001GL014270, 2002.

Liemohn, M. W., Ridley, A. J., Kozyra, J. U., Gallagher, D. L., Thomsen, M. F., Henderson, M. G., Denton, M. H., Brandt, P. C., and Goldstein, J.: Analyzing electric field morphology through data-model comparisons of the GEM IM/S Assessment Challenge events, J. Geophys. Res., 111, A11S11, doi:10.1029/2006JA011700, 2006.

Liemohn, M. W., Zhang, J.-C., Thomsen, M. F., Borovsky, J. E., Kozyra, J. U., and Ilie, R.: Plasma properties of superstorms at geosynchronous orbit: How different are they?, Geophys. Res. Lett., 35, L06S06, doi:10.1029/2007GL031717, 2008.

McIlwain, C. E.: A Kp dependent equatorial electric field model, Adv. Space Res., 6, 187-197, 1986.

Milillo, A., Orsini, S., and Daglis, I. A.: Empirical model of proton fluxes in the equatorial inner magnetosphere: 1. Development, J. Geophys. Res., 106, 25713-25730, 2001.

Ohtani, S., Nose, M., Rostoker, G., Singer, H., Lui, A. T. Y., and 
Nakamura, M.: Storm-substorm relationship: Contribution of the tail current to Dst, J. Geophys. Res., 106, 21199-21209, 2001.

Orsini, S., Milillo, A., and Mura, A.: Modeling of the inner magnetospheric time-evolving plasma: An empirical approach based on proton distribution, J. Geophys. Res., 109, A11216, doi:10.1029/2004JA010532, 2004.

Peredo, M., Stern, D. P., and Tsyganenko, N. A.: Are existing magnetospheric models excessively stretched? J. Geophys. Res., 98, 15343-15354, 1993.

Richmond, A. D.: Assimilative mapping of ionospheric electrodynamics, Adv. Space Res., 12, 59-68, 1992.

Ridley, A. J. and Liemohn, M. W.: A model-derived description of the penetration electric field, J. Geophys. Res., 107, 1151, doi:10.1029/2001JA000051, 2002.

Roederer, J. G.: Dynamics of geomagnetically trapped radiation, Springer-Verlag, New York, 36 pp., 1970.

Sarris, T. E, Li, X., Tsaggas, N., and Paschalidis, N.: Modeling energetic particle injections in dynamic pulse fields with varying propagation speeds, J. Geophys. Res., 107, 1033, doi:10.1029/2001JA900166, 2002.

Sckopke, N.: A general relation between the energy of trapped particles and the disturbance field near the Earth, J. Geophys. Res., 71, 3125-3130, 1966.

Spiro, R. W., Harel, M., Wolf, R. A., and Reiff, P. H.: Quantitative Simulation of A Magnetospheric Substorm, 3. Plasmaspheric Electric Fields and Evolution of the Plasmapause, J. Geophys. Res., 86, 2261-2272, doi:10.1029/JA086iA04p02261, 1981.

Stern, D.: The Motion of a Proton in the Equatorial Magnetosphere, J. Geophys. Res., 80, 595-599, 1975.

Sugiura, M. and Kamei, T.: Equatorial Dst Index 1957-1986, IAGA Bulletin No. 40, ISGI, Saint-Maur-des-fosses, France, 1991.

Thomsen, M. F., Borovsky, J. E., Skoug, R. M., and Smith, C. W.: Delivery of cold, dense plasma sheet material into the near-Earth region, J. Geophys. Res., 108, 1151, doi:10.1029/2002JA009544, 2003.

Toffoletto, F., Sazykin, S., Spiro, R., and Wolf, R.: Inner magnetospheric modeling with the Rice Convection Model, Space Sci. Rev., 107, 175-196, 2003.

Tsyganenko, N. A.: A magnetospheric magnetic field model with a warped tail current sheet, Planet. Space Sci., 37, 5-20, 1989.

Tsyganenko, N. A.: Modeling the Earth's magnetospheric magnetic field confined within a realistic magnetopause, J. Geophys. Res., 100, 5599-5612, 1995.
Tsyganenko, N. A.: A model of the near magnetosphere with a dawn-dusk asymmetry: 2. Parameterization and fitting to observations, J. Geophys. Res., 107, 1176, doi:10.1029/2001JA000220, 2002.

Tsyganenko, N. A. and Mukai, T.: Tail plasma sheet models derived from Geotail particle data, J. Geophys. Res., 108, 1136, doi:10.1029/2002JA009707, 2003.

Tsyganenko, N. A. and Sitnov, M. I.: Modeling the dynamics of the inner magnetosphere during strong geomagnetic storms, J. Geophys. Res., 110, A03208, doi:10.1029/2004JA010798, 2005.

Tsyganenko, N. A., Singer, H. J., and Kasper, J. C.: Storm-time distortion of the inner magnetosphere: How severe can it get?, J. Geophys. Res., 108, 1209, doi:10.1029/2002JA009808, 2003.

Volland, H.: A Semiempirical Model of Large-Scale Magnetospheric Electric Fields, J. Geophys. Res., 78, 171-180, 1973.

Weimer, D. R.: A flexible, IMF dependent model of high-latitude electric potentials having "Space Weather" applications, Geophys. Res. Lett., 23, 2549-2552, 1996.

Wolf, R. A., Harel, M., Spiro, R. W., Voigt, G.-H., Reiff, P. H., and Chen, C.-K.: Computer Simulation of Inner Magnetospheric Dynamics for the Magnetic Storm of July 29, 1977, J. Geophys. Res., 87, 5949-5962, doi:10.1029/JA087iA08p05949, 1982.

Zaharia, S., Cheng, C. Z., and Maezawa, K.: 3-D force-balanced magnetospheric configurations, Ann. Geophys., 22, 251-265, doi:10.5194/angeo-22-251-2004, 2004.

Zaharia, S., Thomsen, M. F., Birn, J., Denton, M. H., Jordanova, V. K., and Cheng, C. Z.: Effect of storm-time plasma pressure on the magnetic field in the inner magnetosphere, Geophys. Res. Lett., 32, L03102, doi:10.1029/2004GL021491, 2005.

Zaharia, S., Jordanova, V. K., Thomsen, M. F., and Reeves, G. D.: Self-consistent modeling of magnetic fields and plasmas in the inner magnetosphere: Application to a geomagnetic storm, J. Geophys. Res., 111, A11S14, doi:10.1029/2006JA011619, 2006.

Zheng, Y., Lui, A. T. Y., Fok, M.-C., Anderson, B. J., Brandt, P. C., Immel, T. J., and Mitchell, D. G.: Relationship between Region 2 field-aligned current and the ring current: Model results, J. Geophys. Res., 111, A11S06, doi:10.1029/2006JA011603, 2006.

Zheng, Y., Lui, A. T. Y., and Fok, M.-C.: Effects of plasma sheet properties on storm-time ring current, J. Geophys. Res., 115, A08220, doi:10.1029/2009JA014806, 2010. 